Ronald P. Muraro

EDIS FE630

John W. Hebb

http://edis.ifas.ufl.edu/FE630

\title{
Budgeting Costs and Returns for Indian River Citrus Production, 2004-05
}
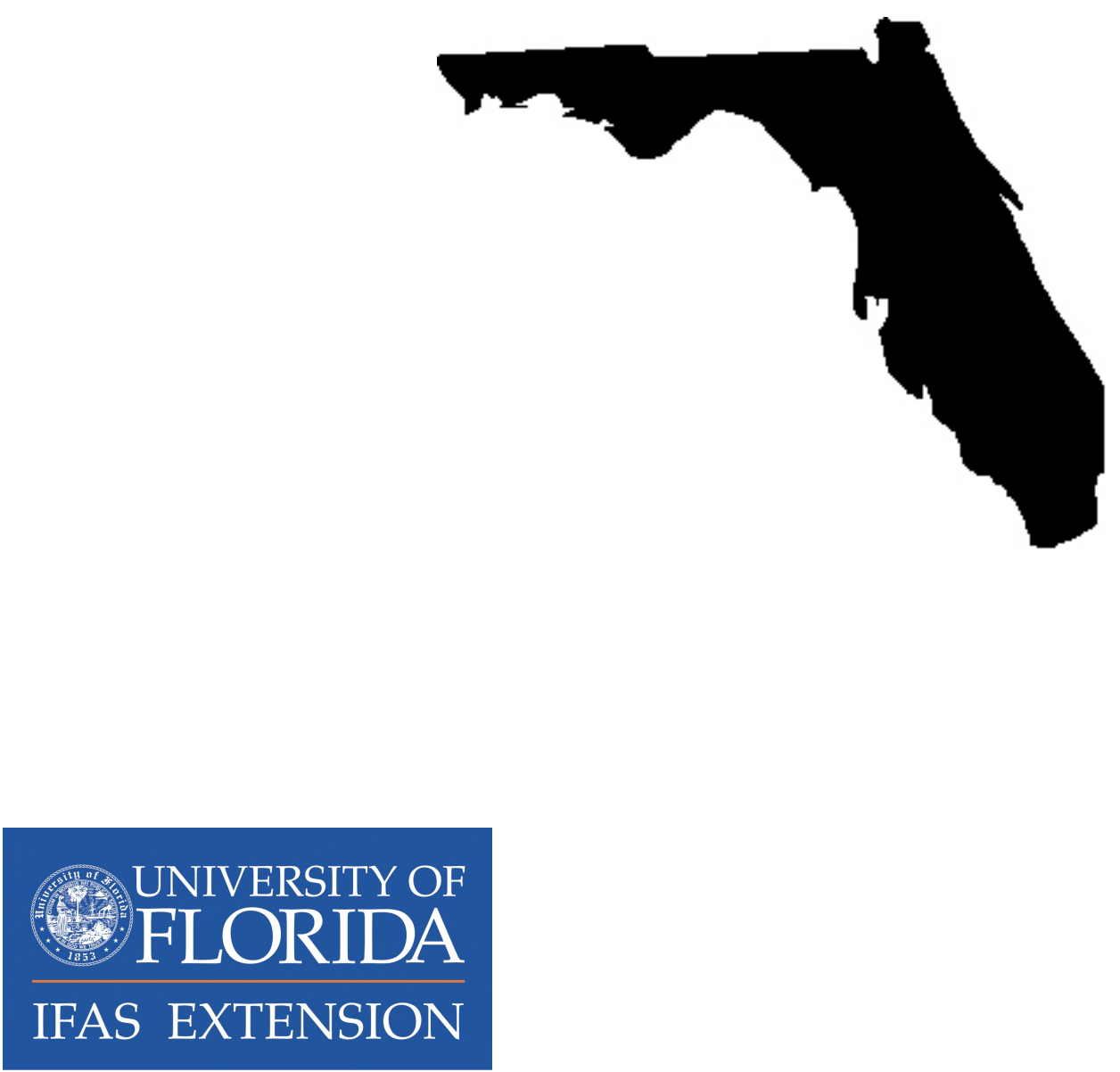

Institute of Food and Agricultural Sciences

Food and Resource Economics Department

Florida Agricultural Experiment Station

Florida Cooperative Extension Service 


\title{
ABSTRACT
}

Estimated costs and returns of growing seedless grapefruit in the Indian River area of Florida are presented for the twenty-second year. The format presented may be used by individual growers to budget costs and returns, utilizing individual data on specific groves.

Key words: citrus, Indian River, budgeting, costs and returns, seedless grapefruit.

NOTE: The Indian River production area refers to the citrus producing counties on Florida's east coast including Brevard, Indian River, Martin, Palm Beach, and St. Lucie counties.

The budgeted cost information presented herein is the most current available. The budget cost items have been revised to reflect current grove practices being used by growers--e.g., chemical mowing, different spray materials and rates of fertilization, microsprinkler irrigation, more reset trees, etc. The 2004-2005 budgets reflect major cost increases in all production inputs: fuel averaged a $22 \%$ increase; fertilizer products increased $15 \%$; chemicals averaged an $8 \%$ increase; and equipment operation costs increased $7 \%$. Along with the increased costs, three major hurricanes (storms) during August and September 2004 resulted in wide tree damage and fruit loss. The Indian River region experienced fruit loss of $70 \%$ to $80 \%$ on red and white grapefruit, respectively. Hamlin orange losses in the Central Florida (ridge) region were $30 \%$ to $40 \%$ with Valencia orange losses between $20 \%$ and $30 \%$. The only citrus growing region that was not significantly affected by the three storms was the Southwest Florida citrus region. As a result of the excessive fruit loss, the per box, per pound solid and per carton costs for the Indian River and Central (ridge) growing regions were substantially higher than in recent years.

The budget costs in this report represent a custom-managed operation. Therefore, all equipment costs are based on the average custom rate costs and a 10 percent handling and supervision charge is added to the material cost.

\begin{abstract}
Although the estimated annual per acre grove costs listed are representative for a mature citrus grove (10+ years old), the grove care costs for a specific grove site may differ depending upon the tree age, tree density and the grove practices performed; e.g., spot herbicide for grass/brush regrowth under trees could add an additional $\$ 16.60$ per acre; Diaprepes control could add $\$ 84.18$ per acre for each foliar application; extensive tree loss due to blight or tristeza could substantially increase the tree replacement and care costs; spray applications to control citrus leafminer and nematicide applications such as Temik (\$127.50/acre) could increase the total cultural costs per acre above the average costs shown in the comparative budgets; travel and set-up costs may vary due to size of the citrus grove and distance from the grove equipment barn and could add $\$ 28.86$ per acre; etc.
\end{abstract}

\section{ACKNOWLEDGEMENTS}

Appreciation is extended to Mrs. Jane Wilson for typing the final draft of this manuscript.

Appreciation is also extended to the citrus growers and production managers of the Indian River citrus production area who provided suggestions for the revision of this manuscript. 


\section{TABLE OF CONTENTS}

$\underline{\text { Page }}$

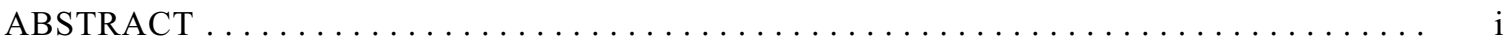

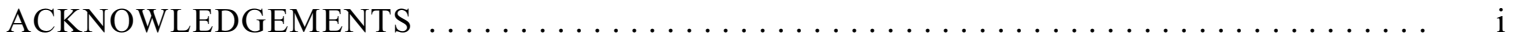

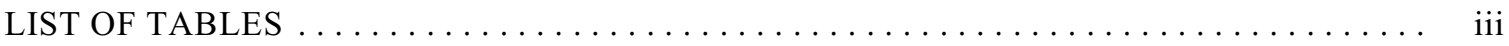

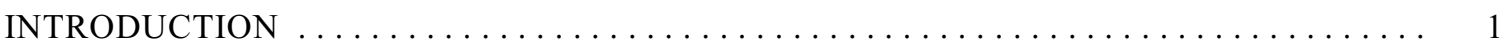

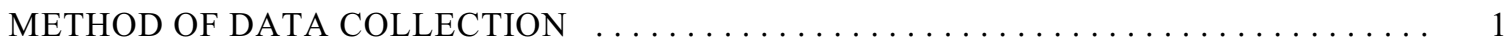

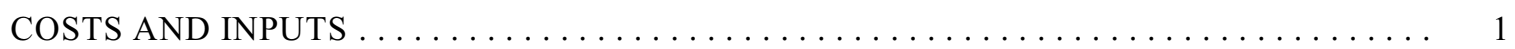

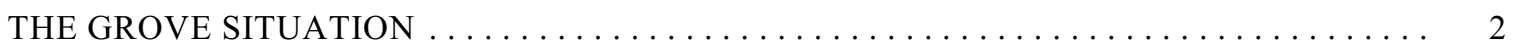

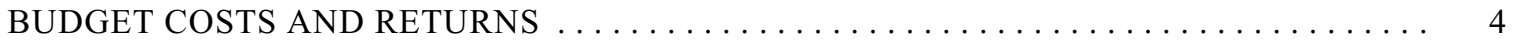

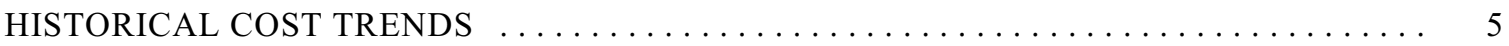

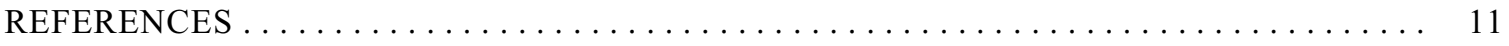

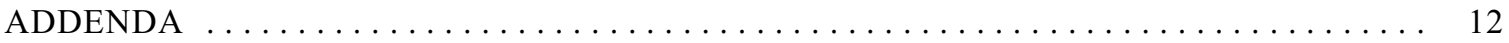

NOTE: The ADDENDA include a Listing of Grove Care Options for Indian River Citrus Production for Both Round Oranges and Grapefruit; 2005 custom rate summary report; cost of establishing a citrus grove; etc. Page 12 is a list of the tables included in the ADDENDA. 


\section{LIST OF TABLES}

$\underline{\text { Table }}$

1 Schedule of production practices and budget items for an Indian River Florida

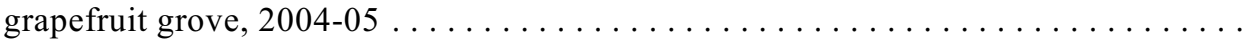

Calculation of normal production per acre, $2004-05 \ldots \ldots \ldots \ldots \ldots \ldots \ldots$

Estimated annual per acre costs and returns for a mature, white seedless grapefruit grove producing for the fresh market, Indian River area, 2004-05 . . . . . . . . . .

Estimated total delivered-in cost for Indian River White grapefruit grown for the processed juice market under three cultural cost programs, 2004-05 . . . . . . . .

Estimated F.O.B. cost for fresh market Indian River White grapefruit, 2004-05 . . .

Estimated annual per acre costs and returns and 5-year average costs and returns for a mature, white seedless grapefruit grove producing citrus for fresh fruit market in the Indian River area, 2000-01-2004-05 . . . . . . . . . . . . . . . . . . . .

$7 \quad$ Estimated annual per acre costs and returns and 5-year average costs and returns (adjusted to 2005 dollars) for a mature, white seedless grapefruit grove producing citrus for fresh fruit market in the Indian River area, 2000-01-2004-05 . . . . . . . . 


\section{BUDGETING COSTS AND RETURNS FOR INDIAN RIVER \\ CITRUS PRODUCTION, 2004-05}

Ronald P. Muraro and John W. Hebb

\section{INTRODUCTION}

Budget analysis provides the basis for many grower decisions. Budget analysis can be used to calculate potential profits from an operation, to determine cash requirements for an operation, and to determine break-even prices. This report presents a budget constructed from current data and serves as a format for growers to analyze costs and returns from their individual records.

The 2004-2005 budgets reflect major cost increases in all production inputs: fuel averaged a $22 \%$ increase; fertilizer products increased $15 \%$; chemicals averaged an $8 \%$ increase; and equipment operation costs increased 7\%. Along with the increased costs, three major hurricanes (storms) during August and September 2004 resulted in wide tree damage and fruit loss. The Indian River region experienced fruit loss of $70 \%$ to $80 \%$ on red and white grapefruit, respectively. Hamlin orange losses in the Central Florida (ridge) region were $30 \%$ to $40 \%$ with Valencia orange losses between $20 \%$ and $30 \%$. The only citrus growing region that was not significantly affected by the three storms was the Southwest Florida citrus region. As a result of the excessive fruit loss, the per box, per pound solid and per carton costs for the Indian River and Central (ridge) growing regions were substantially higher than in recent years.

\section{METHOD OF DATA COLLECTION}

The data presented here were developed by surveying custom operators, input suppliers, growers, and colleagues at both the Indian River Research and Education Center in Ft. Pierce and the Citrus Research and Education Center in Lake Alfred and County Extension Citrus Agents in the Indian River production region. The survey is conducted annually in February and March.

\section{COSTS AND INPUTS}

Costs for various production inputs are those collected from citrus growers as well as the average of the data obtained from annual custom rate, chemical, and fertilizer surveys. Growers' costs are shown in the ADDENDA, Tables 1-A through 7-A. The custom rate costs are shown in Table 8-A and the various chemical and fertilizer costs are shown in Table 9-A and 10-A in the ADDENDA. The

RONALD P. MURARO is a Professor of Food and Resource Economics and Extension Farm Management Economist stationed at the Citrus Research and Education Center, Lake Alfred. JOHN W. HEBB is Extension Agent, Citrus in St. Lucie County, Ft. Pierce. 
budget costs represent a custom-managed operation. Therefore, all equipment costs are based upon the average custom-rate costs and a 10 percent handling and supervision charge is added to the material cost.

Although brand names are used in many of the tables in the ADDENDA, this does not imply endorsement by the University of Florida. It is merely an attempt to depict typical production practices.

All tables have a column reserved for the individual growers to insert data from a particular grove allowing a comparison of the grower's costs with those presented.

\section{THE GROVE SITUATION}

Production practices for an Indian River grapefruit grove are shown in Table 1 with times during the year when they would likely be performed. There are two benefits to developing such a table for an individual grove. First, it shows what work is needed and when, so that operations can be planned well in advance. Second, it can be helpful if an annual cash flow analysis is developed to plan financing. The individual grower may benefit from developing a plan for a particular grove.

Specific production practices vary from grove to grove making it difficult to define a "typical" grove. Many combinations of practices and various tree variety combinations produce acceptable yields and returns. Although the example represents a white seedless grapefruit grove, the cost and return data are designed to be applicable to most grove situations. A grower, realtor, or land appraiser can substitute individual grove costs and expected returns into the budget format and develop a budget for a particular grove. A "your cost" column is appropriately provided for this purpose in subsequent tables.

In the following budget, above average management and cultural practices are assumed. Beyond this general assumption, the following specifics are assumed.

1. A mature (10+ years old), low volume-irrigated grove;

2. Variety is white seedless on sour orange rootstock;

3. Tree loss is 5.0 percent annually;

4. Trees are pulled and replaced when production falls below 50 percent of expected yield;

5. Production is for fresh market;

6. Tree density is 95 trees per acre; and

7. Custom-caretaker is providing grove management.

As a result of tree losses and replacement, the tree ages will vary. The budget reflects the following age distribution and yield for Indian River white seedless grapefruit: 
Table 1.--Schedule of production practices and budget items for an Indian River Florida grapefruit grove, 2004-05

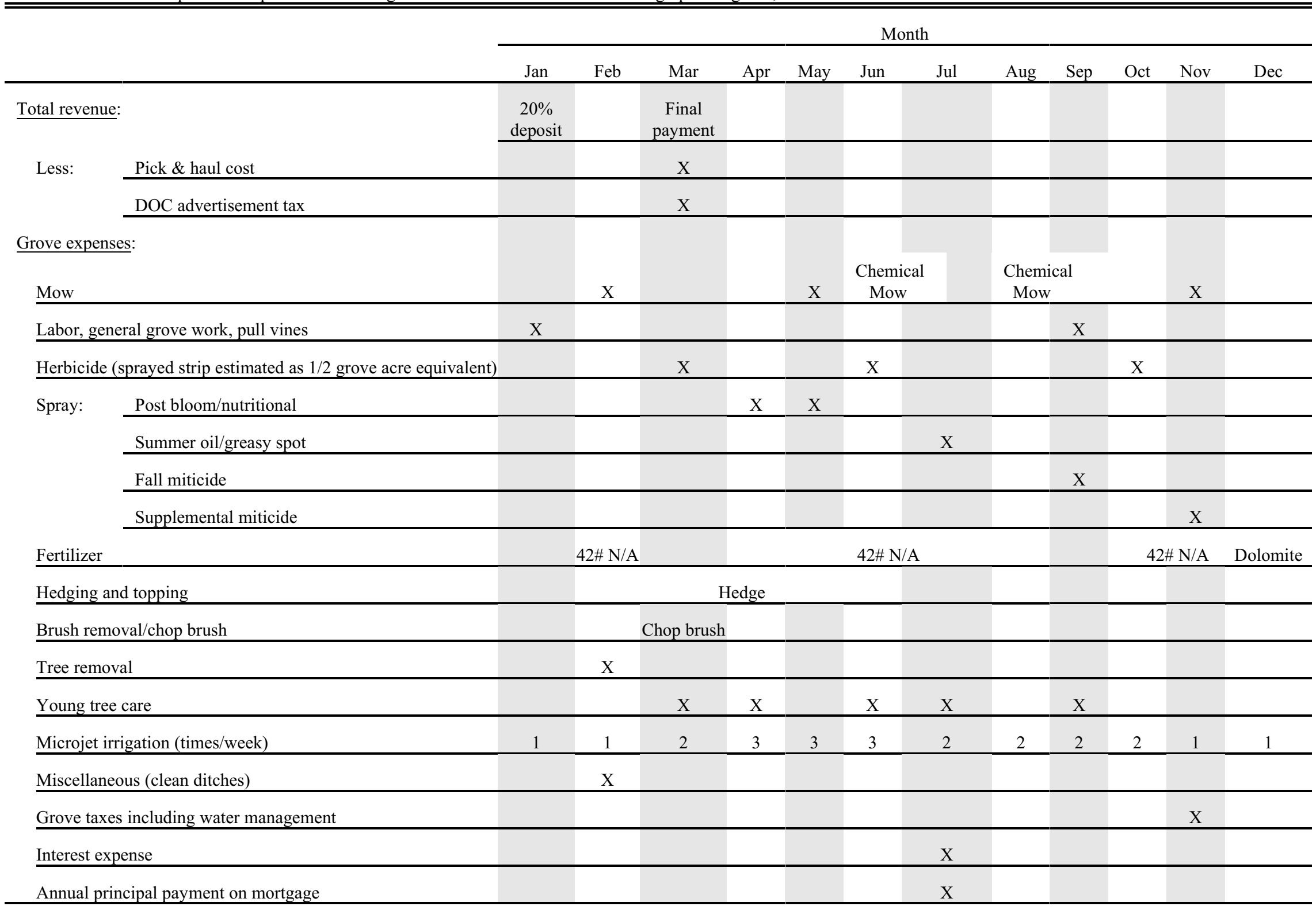

${ }^{a}$ This is a suggested schedule of practices. Actual practices would not necessarily be done on the exact schedule shown here. 


$\begin{array}{rlc}\begin{array}{r}\% \text { of } \\ \text { grove }\end{array} & \text { Tree age and condition } & \begin{array}{c}\text { Yield } \\ \text { boxes/tree }\end{array} \\ 5.0 \% & \text { pulled and reset } & 0.0 \\ 5.0 \% & \text { 1 year old } & 0.0 \\ 5.0 \% & \text { 2 years old } & 0.0 \\ 5.0 \% & \text { 3 years old } & 1.0 \\ 5.0 \% & \text { 4 years old } & 1.7 \\ 55.0 \% & \text { 5-15 years old } & 5.7 \\ 5.0 \% & \text { producing 50\% of expected yield } & 3.5 \\ 15.0 \% & \text { over 15 years } & 7.0\end{array}$

Calculation of normal production per acre is shown in Table 2. Note that the proportion-of-treesby-age column only adds to 85 percent since 15 percent of the trees are non-bearing. The impact of the three hurricanes in 2004 is reflected in a $78.5 \%$ reduction in normal yields.

Table 2.--Calculation of normal production per acre, 2004-05

\begin{tabular}{|c|c|c|c|c|c|c|c|c|c|}
\hline Age of Tree & & & Trees & & & & $\begin{array}{l}\text { Boxes } \\
\text { /tree }\end{array}$ & & $\begin{array}{r}\text { Total } \\
\text { boxes }\end{array}$ \\
\hline & $\begin{array}{l}\text { Total no. } \\
\text { all ages }\end{array}$ & & $\begin{array}{c}\text { Proportion } \\
\text { ea. age }\end{array}$ & & $\begin{array}{c}\text { No. ea. } \\
\text { age }\end{array}$ & & ---- & No. & -------- \\
\hline 3 years & 95 & $\mathrm{x}$ & 0.05 & $=$ & 4.75 & $\mathrm{x}$ & 1.0 & $=$ & 4.8 \\
\hline 4 years & 95 & $\mathrm{x}$ & 0.05 & $=$ & 4.75 & $\mathrm{x}$ & 1.7 & $=$ & 8.1 \\
\hline $5-15$ years & 95 & $\mathrm{x}$ & 0.55 & $=$ & 52.30 & $\mathrm{x}$ & 5.7 & $=$ & 298.1 \\
\hline $\begin{array}{l}\text { Prod. } 50 \% \text { of } \\
\text { exp. yield }\end{array}$ & 95 & $\mathrm{x}$ & 0.05 & $=$ & 4.75 & $\mathrm{x}$ & 3.5 & $=$ & 16.6 \\
\hline Over 15 years & 95 & $\mathrm{x}$ & 0.15 & $=$ & 14.30 & $\mathrm{x}$ & 7.0 & $=$ & $\underline{100.1}$ \\
\hline \multicolumn{8}{|c|}{ Total boxes } & $=$ & $\underline{427.7}$ \\
\hline \multicolumn{9}{|c|}{ Yields adjusted to $21.5 \%$ of normal yields due to three hurricanes in 2004 . } & 92.0 \\
\hline
\end{tabular}

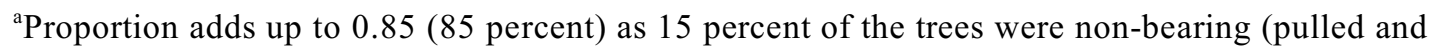
reset, 1 and 2 year old trees).

\section{BUDGET COSTS AND RETURNS}

The estimated budget costs and returns for the Indian River grove situation are shown in Table 3. The budgeted costs represent one possible citrus production program and were selected from the costs shown in the ADDENDA tables. The gross revenue estimates are based on the projected yields in Table 3 and estimated preliminary on-tree prices for the 2004-05 season. Grove establishment and reset costs, alternative cost scenarios, harvesting and packing charges can be found in Tables 11-A through 15-A in the ADDENDA. Also, historical on-tree prices for selected Florida citrus varieties are shown in Table 16-A of the ADDENDA. 
As shown in Table 3, the total revenue for fresh-market white seedless grapefruit is estimated to be $\$ 1,099.40$ per acre. Total specified costs are $\$ 1,195.78$ and are comprised of grove care costs of $\$ 1,147.78$, plus management cost of $\$ 48.00$. Return to land, trees, and ownership, which represents net return above variable costs, was estimated to be a $\$ 96.38$ per acre loss. At 325 and 525 boxes per acre, respectively, the break-even price required to cover grove care costs for seedless white grapefruit range from $\$ 3.54$ to $\$ 2.19$ per box on-tree and $\$ 1.54$ to $\$ 1.25$ per pounds solids delivered-in for eliminations.

Ad valorem taxes, and overhead and administrative costs (such as water drainage district taxes, crop insurance, and other grower assessments) can add up to 12 percent of the total grove care costs. These costs vary from grove to grove depending on age, location, variety of fruit, etc. and should be considered in arriving at a net return to land, trees, and ownership (total return minus total costs). Harvest costs (pick, roadside, and hauling costs) also add to the total fruit cost delivered to either a processing plant or fresh fruit packinghouse. Also, average annual debt payment (principal and interest) may be as high as $\$ 460$ per acre $(\$ 3,900$ average debt per acre @ 10 percent interest amortized over 20 years) which would reduce total available cash for grove expansion or other investment.

Estimated "delivered-in" costs are shown for fresh packed white grapefruit in Table 4. "Delivered-in" costs include grove care costs (Table 3) plus harvesting, regulatory, and grower assessment costs. The "delivered-in" cost is presented as a cost per acre, per box, per carton, and per pound solids. Three possible budget cost scenarios are presented (Refer to Table 13-A): 1) Low Cost Processed Cultural Program; 2) Processed and Reduced Cost Fresh Cultural Program; and 3) Typical/Historical Fresh Fruit Cultural Program. The first scenario represents costs of a cultural program directed toward reducing the expenditures for fruit grown primarily for the processed market. Scenario 2 represents a program using reduced inputs but with production directed at the fresh market. And the third scenario represents typical costs for grove practices which have been performed for citrus grown for the fresh fruit market. Modified herbicide and/or spray and fertilizer programs account for the reduced costs. NOTE: Before modifying a grove management program to reduce costs, an evaluation of the market program (processed or fresh), yield, and specific cultural problems (nutrition, disease, etc.) for the specific grove site should be made. Also, in Table 5, the total estimated F.O.B. cost for fresh packed white grapefruit is shown. The F.O.B. costs are presented for "fresh fruit packout percentage rates" ranging from 50 percent to 100 percent.

\section{HISTORICAL COST TRENDS}

Annual budgets of costs and returns for mature, fresh, white seedless grapefruit in the Indian River area have been developed and published the past four years. Estimated cost and return histories for 2000-01 through 2003-04 along with 2004-05, and a five-year average are presented in Table 6 . The affects of over planting following the 1980s freezes on Florida's annual grapefruit supply has resulted in a fluctuating on-tree price per box. Despite general reduction in operating costs, annual net return to land and trees has decreased over the five-year period. To allow comparisons in current values, these same costs and returns, adjusted to 2005 dollars, are presented in Table 7. 


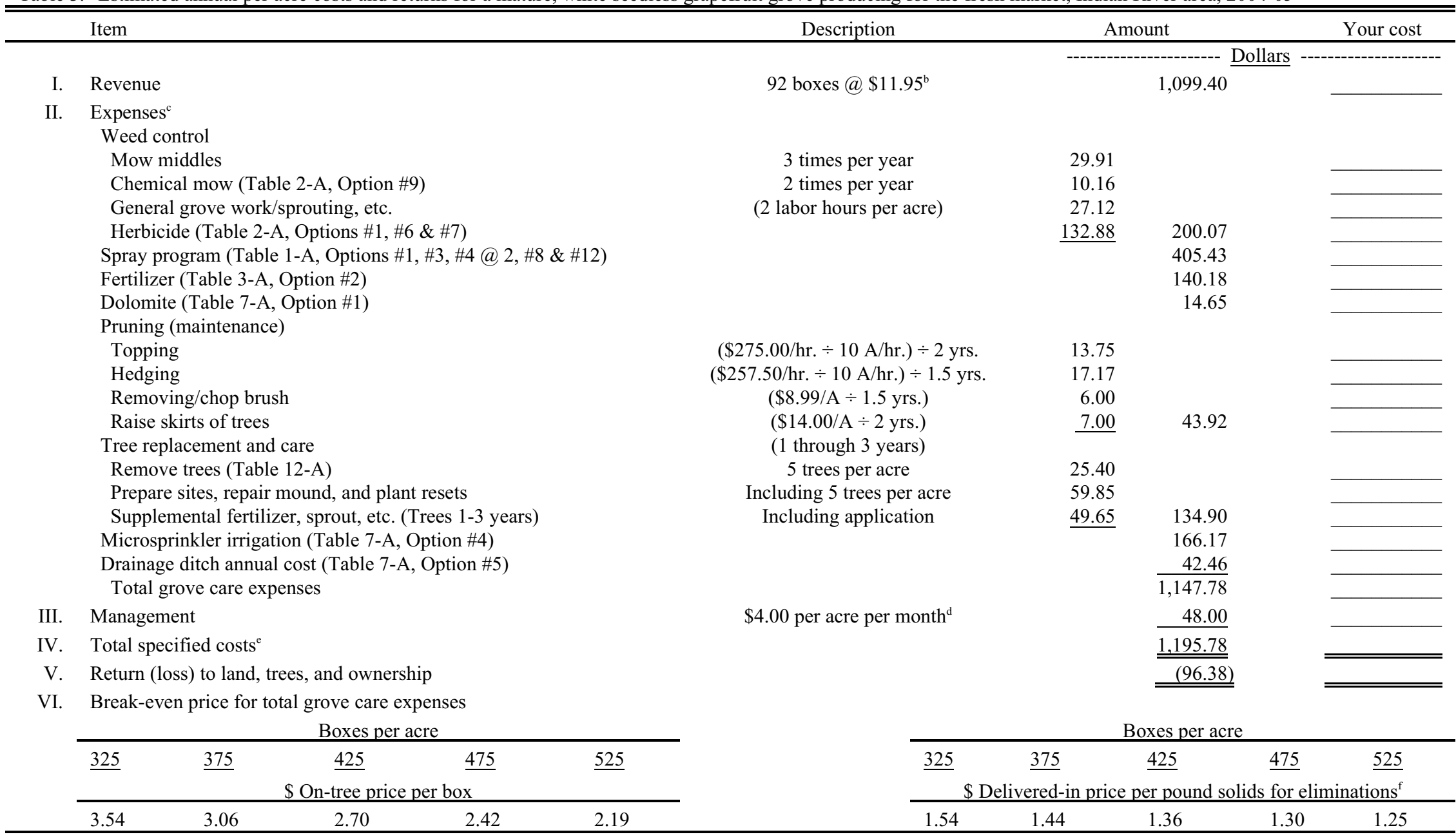

aAlthough the estimated annual per acre grove costs shown in Table 3 are representative for a mature Indian River white seedless grapefruit grove, the grove care costs for a specific grove site may differ depending upon the grove practices performed; e.g., a Temik application would add $\$ 127.50$ per acre; extensive tree loss due to blight or tristeza may double the tree replacement and care costs; travel and set-up costs may vary due to size of citrus grove and distance from grove equipment barn; etc.; truck watering of resets could add another $\$ 7.95$ per acre (average 5 waterings).

${ }^{b}$ On-tree price per box is preliminary; assumes average of all methods of sale (fresh and processed).

${ }^{\circ}$ Assumes material custom applied; therefore, a 10 percent handling and supervision charge is added to material cost.

${ }^{\mathrm{d}}$ Other methods to estimate a management cost--e.g., $5 \%$ of gross sales or $10 \%$ of total grove care costs--are used in the industry. Other methods will give a different return to land and trees than reported here.

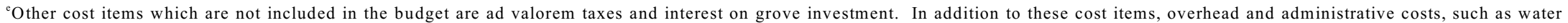
drainage/district taxes, crop insurance, and other grower assessments, can add up to 12 percent to the total grove care costs. These costs vary from grove to grove depending on age, location, and time of purchase or grove establishment.

${ }^{\mathrm{f}}$ Assumes 4.7 pounds solids per box, $\$ 2.63$ pick and haul cost per box (includes spot picking and fruit drenching plus D.O.C. $\$ 0.25$ advertising tax and canker decontamination costs), $\$ 0.55$ per box handling through packinghouse, and $\$ 0.45$ per box delivery to processing plant. 
Table 4. Estimated total delivered-in cost for Indian River White grapefruit grown for the fresh/processed market under three cultural cost programs, 2004-05

\begin{tabular}{|c|c|c|c|c|c|c|c|c|c|}
\hline \multirow[t]{2}{*}{$\begin{array}{l}\text { Represents a mature }(10+\text { years old }) \\
\text { Indian River White Grapefruit Grove }\end{array}$} & \multicolumn{3}{|c|}{$\begin{array}{c}\text { Processed White Grapefruit } \\
\text { Low Cost } \\
\text { Cultural Program }\end{array}$} & \multicolumn{3}{|c|}{$\begin{array}{c}\text { Fresh Packed White Grapefruit } \\
\text { Reduced Cost } \\
\text { Cultural Program }\end{array}$} & \multicolumn{3}{|c|}{$\begin{array}{c}\text { Fresh Packed White Grapefruit } \\
\text { Typical/Historical } \\
\text { Cultural Program }\end{array}$} \\
\hline & $\$ /$ Acre & $\$ /$ Box & $\$ /$ P.S. & $\$ /$ Acre & $\$ /$ Box & $\$ /$ Carton & $\$ /$ Acre & $\$ /$ Box & \$/Carton \\
\hline Total Production/Cultural Costs & $\$ 850.02$ & $\$ 9.239$ & $\$ 1.9658$ & $\$ 1,124.82$ & $\$ 2.528$ & $\$ 1.2638$ & $\$ 1,147.78$ & $\$ 12.476$ & $\$ 6.2379$ \\
\hline Interest on Operating (Cultural) Costs & 23.38 & 0.254 & 0.0541 & 56.24 & 0.126 & 0.0632 & 57.39 & 0.624 & 0.3119 \\
\hline Management Costs & 48.00 & 0.522 & 0.1110 & 48.00 & 0.522 & 0.2609 & 48.00 & 0.522 & 0.2609 \\
\hline \multicolumn{10}{|l|}{ Taxes/Regulatory Costs: } \\
\hline Property Tax/Water Management Tax & 47.04 & 0.511 & 0.1088 & 44.80 & 0.487 & 0.2435 & 44.80 & 0.487 & 0.2435 \\
\hline Water Drainage District Tax & 63.00 & 0.685 & 0.1457 & 60.00 & 0.652 & 0.3261 & 60.00 & 0.652 & 0.3261 \\
\hline Fly Protocol Cost & - & - & - & 54.73 & 0.595 & 0.2974 & 52.13 & 0.567 & 0.2833 \\
\hline Canker Decontamination Costs & 6.18 & 0.067 & $\underline{0.0143}$ & 6.18 & $\underline{0.067}$ & $\underline{0.0336}$ & 6.18 & $\underline{0.067}$ & $\underline{0.0336}$ \\
\hline Total Taxes/Regulatory Costs & 116.22 & $\underline{1.263}$ & $\underline{0.2688}$ & 165.71 & $\underline{1.801}$ & $\underline{0.9006}$ & 163.11 & $\underline{1.773}$ & $\underline{0.8865}$ \\
\hline Total Direct Grower Costs & $\$ 1,037.62$ & $\$ 11.278$ & $\$ 2.3997$ & $\$ 1,394.77$ & $\$ 4.977$ & $\$ 2.4885$ & $\$ 1,416.28$ & $\$ 15.394$ & $\$ 7.6972$ \\
\hline Interest on Average Capital Investment Costs & 321.22 & $\underline{3.491}$ & $\underline{1.7457}$ & 321.22 & $\underline{3.491}$ & $\underline{1.7457}$ & 321.22 & $\underline{3.491}$ & $\underline{1.7457}$ \\
\hline Total Grower Costs & $\$ 1,358.83$ & $\$ 14.770$ & $\$ 4.1454$ & $\$ 1,715.99$ & $\$ 8.468$ & $\$ 4.2342$ & $\$ 1,737.49$ & $\$ 18.886$ & $\$ 9.4429$ \\
\hline \multicolumn{10}{|l|}{ Harvesting and Assessment Costs: } \\
\hline $\begin{array}{l}\text { Pick/Spot Pick, Roadside \& Haul and } \\
\text { Canker Decontamination }\end{array}$ & 191.54 & 2.082 & 0.4430 & 218.41 & 2.374 & 1.1870 & 218.41 & 2.374 & 1.1870 \\
\hline Fruit Drenching (Fresh) & - & - & - & 17.02 & 0.185 & 0.0925 & 17.02 & 0.185 & 0.0925 \\
\hline DOC Assessment & 22.08 & $\underline{0.240}$ & $\underline{0.0511}$ & 23.00 & $\underline{0.250}$ & $\underline{0.1250}$ & 23.00 & $\underline{0.250}$ & $\underline{0.1250}$ \\
\hline Total Harvesting and Assessment Costs & 213.62 & 2.322 & 0.4940 & 258.43 & 2.809 & 1.4045 & 258.43 & 2.809 & 1.4045 \\
\hline Total Delivered-In Cost & $\$ \underline{\underline{1,572.45}}$ & $\$ \underline{\underline{17.092}}$ & $\$ \underline{\underline{4.6394}}$ & $\$ \underline{\underline{\underline{1,974.41}}}$ & $\$ \underline{\underline{11.277}}$ & $\$ \underline{\underline{\underline{5.6387}}}$ & $\$ 1,995.92$ & $\$ \underline{\underline{\underline{21.695}}}$ & $\$ 10.8474$ \\
\hline $\begin{array}{l}\text { Two cartons per box } \\
\text { P.S.=Pound Solids } \\
\text { Yield: } 92 \text { boxes/acre @ } 4.7 \text { P.S. per box } \\
95 \text { trees per acre }\end{array}$ & \multicolumn{3}{|c|}{$\begin{array}{l}\text { Refer to cultural program } \\
\text { shown on Table 13-A. }\end{array}$} & \multicolumn{3}{|c|}{$\begin{array}{l}\text { Refer to cultural program shown } \\
\text { in Table 13-A. }\end{array}$} & \multicolumn{3}{|c|}{$\begin{array}{c}\text { Refer to cultural program shown } \\
\text { in Table } 3 .\end{array}$} \\
\hline
\end{tabular}


8

Table 5.--Estimated F.O.B. cost for fresh market Indian River White grapefruit, 2004-05

\begin{tabular}{|c|c|c|c|c|c|c|c|c|c|}
\hline & \multicolumn{3}{|c|}{\begin{tabular}{|cc} 
Percent Packout & $50.00 \%$ \\
Box Yield Per Acre & 445 \\
Per & \\
Packed &
\end{tabular}} & \multicolumn{3}{|c|}{\begin{tabular}{|cc} 
Percent Packout $\quad 60.00 \%$ \\
Box Yield Per Acre & 445 \\
Per \\
Packed
\end{tabular}} & \multicolumn{3}{|c|}{ 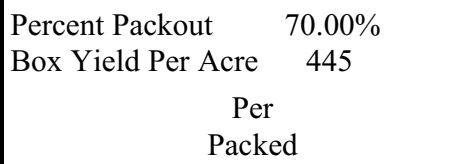 } \\
\hline & Per Acre & Box & Per Carton & Per Acre & & Per Carton & Per Acre & Box & Per Carton \\
\hline $\begin{array}{c}\text { Total Production/ } \\
\text { Cultural Costs }\end{array}$ & $\$ 1,147.78$ & $\$ 5.159$ & $\$ 2.5793$ & $\$ 1,147.78$ & $\$ 4.299$ & $\$ 2.1494$ & $\$ 1,147.78$ & $\$ 3.685$ & $\$ 1.8423$ \\
\hline $\begin{array}{l}\text { Interest on Operating } \\
\text { (Cultural) Costs }\end{array}$ & 57.39 & 0.258 & 0.1290 & 57.39 & 0.215 & 0.1075 & 57.39 & 0.184 & 0.0921 \\
\hline Management & 48.00 & 0.216 & 0.1079 & 48.00 & 0.180 & 0.0899 & 48.00 & 0.154 & 0.0770 \\
\hline Taxes/Regulatory & 163.11 & 0.733 & 0.3665 & 163.11 & 0.611 & 0.3054 & 163.11 & 0.524 & 0.2618 \\
\hline $\begin{array}{l}\text { Interest on Average } \\
\text { Capital Investment }\end{array}$ & 321.22 & 1.444 & 0.7218 & 321.22 & 1.203 & 0.6015 & 321.22 & 1.031 & 0.5156 \\
\hline $\begin{array}{l}\text { Harvesting (Pick/Spot Pick, } \\
\text { Haul, DOC Tax, Etc.) }\end{array}$ & $\underline{1,250.01}$ & $\underline{5.618}$ & $\underline{2.8090}$ & $\underline{1,250.01}$ & $\underline{4.682}$ & $\underline{2.3408}$ & $\underline{1,250.01}$ & $\underline{4.013}$ & $\underline{2.0064}$ \\
\hline Total Delivered-In Cost & $\$ 2,987.50$ & $\$ 13.427$ & $\$ 6.7135$ & $\$ 2,987.50$ & $\$ 11.189$ & $\$ 5.5946$ & $\$ 2,987.50$ & $\$ 9.591$ & $\$ 4.7953$ \\
\hline Packing \& Selling (Export) & $1,642.05$ & 7.380 & 3.6900 & $1,970.46$ & 7.380 & 3.6900 & $2,298.87$ & 7.380 & 3.6900 \\
\hline $\begin{array}{l}\text { Net Fresh Eliminations } \\
\text { Costs }^{\mathrm{a}}\end{array}$ & $\underline{-1,743.51}$ & $\underline{-7.836}$ & $\underline{-3.9180}$ & $\underline{-1,394.81}$ & $\underline{-5.224}$ & $\underline{-2.6120}$ & $\underline{-1,046.11}$ & $\underline{-3.358}$ & $\underline{-1.6791}$ \\
\hline \multirow[t]{2}{*}{ Total F.O.B. Costs } & $\$ 2,886.04$ & $\$ \underline{\underline{12.971}}$ & $\$ \underline{\underline{6.4855}}$ & $\$ \underline{\underline{3,563.15}}$ & $\$ \underline{\underline{13.345}}$ & $\$ \underline{\underline{6.6726}}$ & $\$ \underline{\underline{4,240.26}}$ & $\$ 13.612$ & $\$ \underline{\underline{6.8062}}$ \\
\hline & $\begin{array}{l}\text { Percent Pack } \\
\text { Box Yield P } \\
\text { Per Acre }\end{array}$ & $\begin{array}{l}80 \\
\text { Acre } 4 \\
\text { Per } \\
\text { Packed } \\
\text { Box }\end{array}$ & Per Carton & $\begin{array}{l}\text { Percent Pack } \\
\text { Box Yield P }\end{array}$ & $\begin{array}{l}\text { ut } \quad 90 \\
\text { Acre } \\
\text { Per } \\
\text { Packed } \\
\text { Box }\end{array}$ & Per Carton & \multicolumn{3}{|c|}{$\begin{array}{c}\text { Percent Packout } \quad 100.00 \% \\
\text { Box Yield Per Acre } \quad 445 \\
\text { Per } \\
\text { Packed }\end{array}$} \\
\hline $\begin{array}{l}\text { Total Production/ } \\
\text { Cultural Costs }\end{array}$ & $\$ 1,147.78$ & $\$ 3.224$ & $\$ 1.6121$ & $\$ 1,147.78$ & $\$ 2.866$ & $\$ 1.4329$ & $\$ 1,147.78$ & $\$ 2.579$ & $\$ 1.2896$ \\
\hline $\begin{array}{l}\text { Interest on Operating } \\
\text { (Cultural) Costs }\end{array}$ & 57.39 & 0.161 & 0.0806 & 57.39 & 0.143 & 0.0716 & 57.39 & 0.129 & 0.0645 \\
\hline Management & 48.00 & 0.135 & 0.0674 & 48.00 & 0.120 & 0.0599 & 48.00 & 0.108 & 0.0539 \\
\hline Taxes/Regulatory & 163.11 & 0.458 & 0.2291 & 163.11 & 0.407 & 0.2036 & 163.11 & 0.367 & 0.1833 \\
\hline $\begin{array}{l}\text { Interest on Average } \\
\text { Capital Investment }\end{array}$ & 321.22 & 0.902 & 0.4511 & 321.22 & 0.802 & 0.4010 & 321.22 & 0.722 & 0.3609 \\
\hline $\begin{array}{l}\text { Harvesting (Pick/Spot Pick, } \\
\text { Haul, DOC Tax, Etc.) }\end{array}$ & $\underline{1,250.01}$ & $\underline{3.511}$ & $\underline{1.7556}$ & $\underline{1,250.01}$ & $\underline{3.121}$ & $\underline{1.5606}$ & $\underline{1,250.01}$ & $\underline{2.809}$ & $\underline{1.4045}$ \\
\hline Total Delivered-In Cost & $\$ 2,987.50$ & $\$ 8.392$ & $\$ 4.1959$ & $\$ 2,987.50$ & $\$ 7.459$ & $\$ 3.7297$ & $\$ 2,987.50$ & $\$ 6.713$ & $\$ 3.3567$ \\
\hline Packing \& Selling (Export) & $2,627.28$ & 7.380 & 3.6900 & $2,955.69$ & 7.380 & 3.6900 & $3,284.10$ & 7.380 & 3.6900 \\
\hline $\begin{array}{l}\text { Net Fresh Eliminations } \\
\text { Costs }^{\mathrm{a}}\end{array}$ & -697.40 & $\underline{-1.959}$ & $\underline{-0.9795}$ & -348.70 & $\underline{-0.871}$ & $\underline{-0.4353}$ & 0.00 & $\underline{0.000}$ & $\underline{0.0000}$ \\
\hline Total F.O.B. Costs & $\$ 4,917.38$ & $\$ 13.813$ & $\$ 6.9064$ & $\$ 5,594.49$ & $\$ 13.969$ & $\$ 6.9844$ & $\$ \underline{\underline{6,271.60}}$ & $\$ 14.093$ & $\$ 7.0467$ \\
\hline
\end{tabular}

a "Net Eliminations Cost" equals the average yield of 4.70 pound solids per box times $\$ 1.88$ per pound solids less packinghouse elimination charge and cannery hauling charge of $\$ 1.00$ per box. 
Table 6.--Estimated annual per acre costs and returns for a mature, white seedless grapefruit grove producing citrus for fresh fruit market in the Indian River area, 2000-01-2004-05

\begin{tabular}{|c|c|c|c|c|c|c|}
\hline Year & $\begin{array}{c}\text { On-tree } \\
\text { price/box }{ }^{\mathrm{a}}\end{array}$ & Yield & $\begin{array}{c}\text { Gross } \\
\text { revenue }\end{array}$ & $\begin{array}{c}\text { Total grove } \\
\text { care expenses }\end{array}$ & $\begin{array}{c}\text { Total specified } \\
\text { costs }^{\mathrm{e}}\end{array}$ & $\begin{array}{l}\text { Net return to land, } \\
\text { trees, and ownership }\end{array}$ \\
\hline & & & \multicolumn{4}{|c|}{ 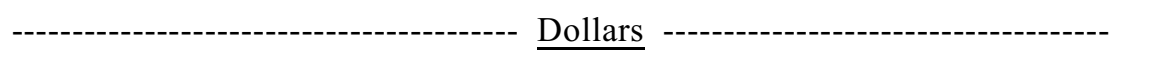 } \\
\hline $2000-01$ & $\$ 2.15$ & $425^{\mathrm{c}}$ & 913.75 & 974.46 & $1,022.46$ & $(108.71)$ \\
\hline $2001-02$ & $\$ 1.95$ & $417^{\mathrm{d}}$ & 813.15 & $1,008.77$ & $1,056.77$ & $(243.62)$ \\
\hline $2002-03$ & $\$ 2.08$ & $417^{\mathrm{d}}$ & 867.36 & $1,024.54$ & $1,072.54$ & $(205.18)$ \\
\hline $2003-04$ & $\$ 1.88$ & 445 & 836.60 & $1,041.13$ & $1,089.13$ & $(252.53)$ \\
\hline $2004-05$ & $\$ 11.95^{b}$ & $92^{\mathrm{c}}$ & $1,099.40$ & $1,147.78$ & $1,195.78$ & $(96.38)$ \\
\hline
\end{tabular}

${ }^{\mathrm{a}}$ On-tree prices for all sales methods as reported by the Florida Agricultural Statistics Service.

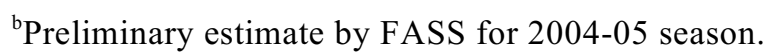

${ }^{\mathrm{c}}$ The severe drought affected yields for the 2001-02 season and three hurricanes in 2004 reduced yields by $78.5 \%$.

${ }^{\mathrm{d}}$ Increased tree loss due to citrus tristeza virus reduced yields.

${ }^{\mathrm{e}} \mathrm{A}$ management cost of $\$ 4.00$ per acre per month is included. Fixed costs such as taxes, debt service, and crop insurance are not included. 
Table 7.--Estimated annual per acre costs and returns (adjusted to 2005 dollars) for a mature, white seedless grapefruit grove producing citrus for fresh fruit market in the Indian River area, 2000-01-2004-05

\begin{tabular}{|c|c|c|c|c|c|c|}
\hline Year & $\begin{array}{c}\text { Inflation } \\
\text { factor index }\end{array}$ & $\begin{array}{l}\text { Adjusted } \\
\text { on-tree } \\
\text { price/box }\end{array}$ & Yield & $\begin{array}{c}\text { Gross } \\
\text { revenue }\end{array}$ & $\begin{array}{c}\text { Total specified } \\
\operatorname{costs}^{\mathrm{b}}\end{array}$ & $\begin{array}{l}\text { Net return to land, } \\
\text { trees, and ownership }\end{array}$ \\
\hline & & & & ------. & Dollars & ----------------------- \\
\hline 2000-01 & 117.9 & $\$ 2.54$ & 425 & $1,079.50$ & $1,205.48$ & (125.98) \\
\hline 2001-02 & 120.7 & $\$ 2.36$ & 417 & 984.12 & $1,275.53$ & $(291.41)$ \\
\hline 2002-03 & 114.6 & $\$ 2.39$ & 417 & 996.63 & $1,229.13$ & $(232.50)$ \\
\hline 2003-04 & 107.9 & $\$ 2.03$ & 445 & 903.35 & $1,175.18$ & $(271.83)$ \\
\hline 2004-05 & 100.0 & $\$ 11.95$ & 92 & $1,099.40$ & $1,195.78$ & (96.38) \\
\hline
\end{tabular}

${ }^{a}$ Producer price index for each year adjusted to 2005 prices $(2005=100)$, with 2005 consumer price index estimated to be 158.2 . Producer price index for other years are: $2001=134.2 ; 2002=131.1 ; 2003=138.1 ;$ and $2004=146.7$.

${ }^{\mathrm{b}} \mathrm{A}$ management cost of $\$ 4.00$ per acre per month is included. Fixed costs such as taxes, debt service, and crop insurance are not included. (Refer to Table 6.) 


\section{REFERENCES}

1. Preliminary Summary 2004-05. Florida Agricultural Statistics Service. Florida Agricultural Statistics. September 2005.

2. Muraro, Ronald P. "A Listing of Estimated Comparative Indian River Citrus Production Costs Per Acre for 2004-05." Lake Alfred Citrus Research and Education Center (CREC) Report. Lake Alfred, FL: August 2005.

3. _ Estimated Cost of Planting and Maintaining a Reset Citrus Tree through Three Years of Age." Lake Alfred CREC Report. Lake Alfred, FL: July 2004.

4. ___ "A Listing of 2005 Custom Rates Reported by Twenty-five Indian River and South Florida Citrus Caretakers." Lake Alfred CREC Report. Lake Alfred, FL: July 2005.

5. Savage, Zach. Citrus Yields Per Tree Age. Univ. of Fla. Agr. Ext. Ser. 60-8. Gainesville: 1960.

6. Timmer, L. W. (Ed.). 2005 Florida Citrus Pest Management Guide. Univ. of Fla. Coop. Ext. Svc. SP 43. Gainesville: Jan. 2005. 150 pp.

7. Tucker, D. P. H., A. K. Alva, L. K. Jackson, and T. A. Wheaton (Eds.). Nutrition of Florida Citrus Trees. Univ. of Fla. Coop. Ext. Svc. SP 169. Gainesville: 1995. 61 pp. 
ADDENDA: Listing of Grove Care Options for Indian River Citrus Production for Both Round Oranges and Grapefruit ${ }^{\mathrm{a}}$

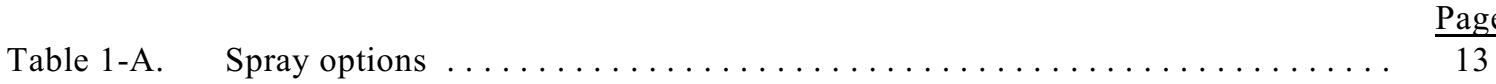

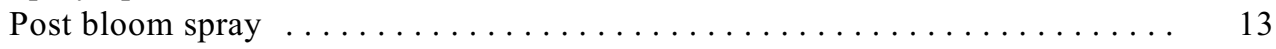

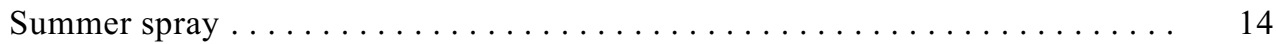

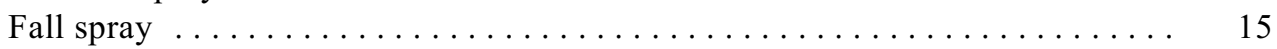

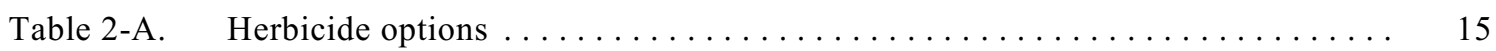

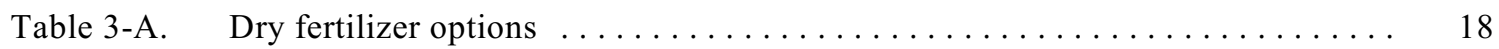

Table 4-A. Liquid fertilizer (Double boom application) $\ldots \ldots \ldots \ldots \ldots$

Table 5-A. Nematicides options $\ldots \ldots \ldots \ldots \ldots \ldots \ldots \ldots \ldots \ldots$

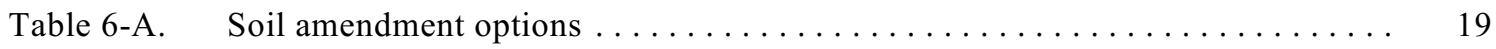

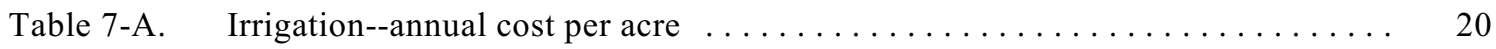

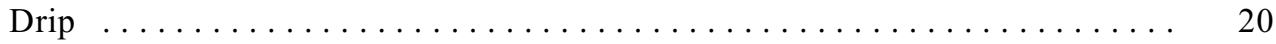

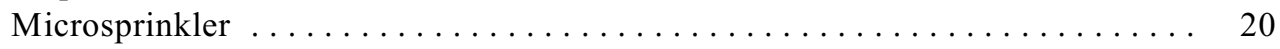

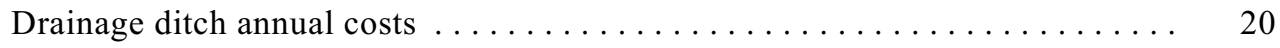

Table 8-A. A listing of 2005 custom rates reported by fifteen Indian River and South

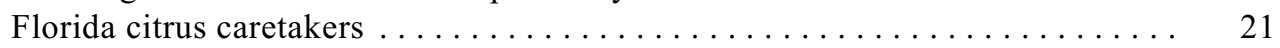

Table 9-A. 2005 summary of average chemical price estimates $\ldots \ldots \ldots \ldots$

Table 10-A. 2005 summary of average fertilizer price estimates $\ldots \ldots \ldots \ldots$

Table 11-A. A listing of estimated comparative Indian River citrus production costs per

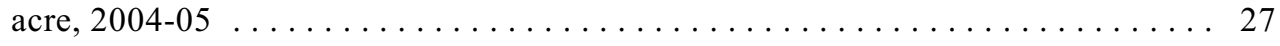

Table 12-A. Estimated cost of planting and maintaining a reset citrus tree through three

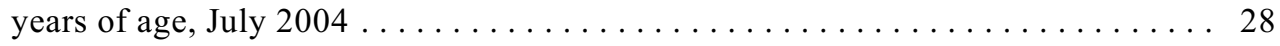

Table 13-A. Estimated average picking, roadsiding and hauling charges for Florida citrus,

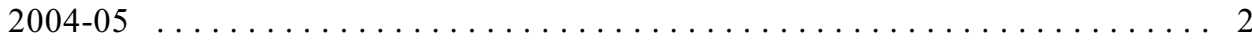

Table 14-A. Estimated average packing charges for Florida citrus, 2004-05 . . . . . . 30

Table 15-A. Historic prices for selected citrus varieties $\ldots \ldots \ldots \ldots \ldots \ldots$

Table 16-A. Debt which can be supported per $\$ 1,000.00$ annual payment capacity $\ldots \ldots \ldots 32$

Abbreviations for important chemicals are:

$\begin{array}{lll}\mathrm{Cu}=\text { Copper } & \mathrm{Mg}=\text { Magnesium } & \mathrm{N}=\text { Nitrogen } \\ \mathrm{Fe}=\text { Iron } & \mathrm{Mn}=\text { Manganese } & \mathrm{Zn}=\text { Zinc }\end{array}$

aThe costs in the ADDENDA represent a custom managed operation. Therefore, all equipment costs are based upon the average custom rate costs and a 10 percent handling and supervision charge is added to the material cost. 
Table 1-A.--Spray options

POST BLOOM SPRAY

\begin{tabular}{|c|c|c|c|c|}
\hline \multirow[t]{8}{*}{ Spray Option \#1 } & $\underline{\text { Materials/Ingredients }}$ & $\begin{array}{l}\text { Amount } \\
\text { /Acre } \\
\end{array}$ & $\underline{\text { Cost/Acre }}$ & $\begin{array}{c}\text { Your } \\
\text { Cost/Acre } \\
\end{array}$ \\
\hline & Micromite & $1.25 \mathrm{lbs}$ & $\$ 42.65$ & \\
\hline & Oil 97+\% & 5 gals & 12.30 & \\
\hline & $\mathrm{Cu}(50 \%$ metallic $)$ & $7 \mathrm{lbs}$ & 10.92 & \\
\hline & $\mathrm{Zn}$ & $5 \mathrm{lbs}$ & 4.60 & \\
\hline & $\mathrm{Mn}$ & $10 \mathrm{lbs}$ & 3.60 & \\
\hline & $\begin{array}{l}\text { Ground Application } \\
\text { (PTO driven airblast) }\end{array}$ & 250 gals & $\underline{32.92}$ & \\
\hline & Total per Application & & $\$ \underline{\underline{106.99}}$ & \\
\hline \multirow[t]{6}{*}{ Spray Option \#2 } & $\underline{\text { Materials/Ingredients }}$ & $\begin{array}{l}\text { Amount } \\
\text { /Acre } \\
\end{array}$ & $\underline{\text { Cost/Acre }}$ & $\begin{array}{c}\text { Your } \\
\text { Cost/Acre } \\
\end{array}$ \\
\hline & Agri-Mek & $10 \mathrm{ozs}$ & $\$ 48.60$ & \\
\hline & $\mathrm{Cu}(50 \%$ metallic $)$ & $10 \mathrm{lbs}$ & 15.60 & \\
\hline & Oil $97+\%$ & 3 gals & 7.38 & \\
\hline & $\begin{array}{l}\text { Ground Application } \\
\text { (Curtec sprayer) }\end{array}$ & $25 \mathrm{GPA}$ & $\underline{22.00}$ & \\
\hline & Total per Application & & $\$ \underline{\underline{93.58}}$ & \\
\hline \multirow[t]{5}{*}{ Spray Option \#3 } & $\underline{\text { Materials/Ingredients }}$ & $\begin{array}{l}\text { Amount } \\
\text { /Acre } \\
\end{array}$ & $\underline{\text { Cost/Acre }}$ & $\begin{array}{c}\text { Your } \\
\text { Cost/Acre } \\
\end{array}$ \\
\hline & $\mathrm{Cu}(50 \%$ metallic $)$ & $7 \mathrm{lbs}$ & $\$ 10.92$ & \\
\hline & Oil $97+\%$ & 5 gals & 12.30 & \\
\hline & $\begin{array}{l}\text { Ground Application } \\
\quad \text { (PTO driven airblast) }\end{array}$ & 250 gals & $\underline{32.92}$ & \\
\hline & Total per Application & & $\$ \underline{\underline{56.14}}$ & \\
\hline \multirow[t]{4}{*}{ Spray Option \#4 } & $\underline{\text { Materials/Ingredients }}$ & $\begin{array}{l}\text { Amount } \\
\text { /Acre } \\
\end{array}$ & $\underline{\text { Cost/Acre }}$ & $\begin{array}{c}\text { Your } \\
\text { Cost/Acre } \\
\end{array}$ \\
\hline & $\mathrm{Cu}(50 \%$ metallic $)$ & $7 \mathrm{lbs}$ & $\$ 10.92$ & \\
\hline & $\begin{array}{l}\text { Ground Application } \\
\text { (PTO driven airblast) }\end{array}$ & 125 gals & $\underline{28.03}$ & \\
\hline & Total per Application & & $\$ \underline{\underline{38.95}}$ & \\
\hline
\end{tabular}


Table 1-A.--Spray options (cont'd.)

$\underline{\text { SUMMER SPRAY }}$

\begin{tabular}{|c|c|c|c|c|}
\hline \multirow[t]{6}{*}{ Spray Option \#5 } & Materials/Ingredients & $\begin{array}{l}\text { Amount } \\
\text { /Acre }\end{array}$ & Cost/Acre & $\begin{array}{c}\text { Your } \\
\text { Cost/Acre } \\
\end{array}$ \\
\hline & Oil $97+\%$ & 5 gals & $\$ 12.30$ & \\
\hline & $\mathrm{Cu}(50 \%$ metallic $)$ & $7 \mathrm{lbs}$ & 10.92 & \\
\hline & Micromite & $1.25 \mathrm{lbs}$ & 42.65 & \\
\hline & $\begin{array}{l}\text { Ground Application } \\
\text { (PTO driven airblast) }\end{array}$ & 250 gals & $\underline{39.92}$ & \\
\hline & Total per Application & & $\$ \underline{\underline{98.59}}$ & \\
\hline \multirow[t]{6}{*}{ Spray Option \#6 } & $\underline{\text { Materials/Ingredients }}$ & $\begin{array}{l}\text { Amount } \\
\text { /Acre } \\
\end{array}$ & $\underline{\text { Cost/Acre }}$ & $\begin{array}{c}\text { Your } \\
\text { Cost/Acre } \\
\end{array}$ \\
\hline & $\mathrm{Cu}(50 \%$ metallic $)$ & $7 \mathrm{lbs}$ & $\$ 10.92$ & \\
\hline & Oil $97+\%$ & 5 gals & 12.30 & \\
\hline & Agri-Mek & $10 \mathrm{ozs}$ & 48.60 & \\
\hline & $\begin{array}{l}\text { Ground Application } \\
\text { (PTO driven airblast) }\end{array}$ & 250 gals & 32.92 & \\
\hline & Total per Application & & $\$ \underline{\underline{104.74}}$ & \\
\hline \multirow[t]{6}{*}{ Spray Option \#7 } & $\underline{\text { Materials/Ingredients }}$ & $\begin{array}{l}\text { Amount } \\
\text { /Acre }\end{array}$ & Cost/Acre & $\begin{array}{c}\text { Your } \\
\text { Cost/Acre } \\
\end{array}$ \\
\hline & $\mathrm{Cu}(50 \%$ metallic $)$ & $7 \mathrm{lbs}$ & $\$ 10.92$ & \\
\hline & Oil $97+\%$ & 10 gals & 24.60 & \\
\hline & Agri-Mek & 5 ozs & 24.30 & \\
\hline & $\begin{array}{l}\text { Ground Application } \\
\text { (PTO driven airblast) }\end{array}$ & 500 gals & $\underline{38.00}$ & \\
\hline & Total per Application & & $\$ \underline{\underline{97.82}}$ & \\
\hline \multirow[t]{6}{*}{ Spray Option \#8 } & Materials/Ingredients & $\begin{array}{l}\text { Amount } \\
\text { /Acre }\end{array}$ & Cost/Acre & $\begin{array}{c}\text { Your } \\
\text { Cost/Acre } \\
\end{array}$ \\
\hline & Enable & $8 \mathrm{oz}$ & $\$ 15.80$ & \\
\hline & Oil $97+\%$ & 5 gals & 12.30 & \\
\hline & Micromite & $1.25 \mathrm{lbs}$ & 42.65 & \\
\hline & $\begin{array}{l}\text { Ground Application } \\
\text { (PTO driven airblast) }\end{array}$ & 250 gals & $\underline{32.92}$ & \\
\hline & Total per Application & & $\$ \underline{\underline{103.67}}$ & \\
\hline \multirow[t]{8}{*}{ Spray Option \#9 } & $\underline{\text { Materials/Ingredients }}$ & $\begin{array}{l}\text { Amount } \\
\text { /Acre }\end{array}$ & Cost/Acre & $\begin{array}{c}\text { Your } \\
\text { Cost/Acre } \\
\end{array}$ \\
\hline & $\mathrm{Cu}(50 \%$ metallic $)$ & $7 \mathrm{lbs}$ & $\$ 10.92$ & \\
\hline & Oil $97+\%$ & 5 gals & 12.30 & \\
\hline & $\mathrm{Zn}$ & $5 \mathrm{lbs}$ & 4.60 & \\
\hline & $\mathrm{Mn}$ & $10 \mathrm{lbs}$ & 3.60 & \\
\hline & $\mathrm{B}$ & $0.25 \mathrm{lbs}$ & 1.34 & \\
\hline & $\begin{array}{l}\text { Ground Application } \\
\text { (PTO driven airblast) }\end{array}$ & 250 gals & $\underline{32.92}$ & \\
\hline & Total per Application & & $\$ \underline{65.68}$ & \\
\hline
\end{tabular}


Table 1-A.-Spray options (cont'd.)

SUMMER SPRAY (cont'd.)

Spray Option \#10 Materials/Ingredients

Amount Your

(Scale insects)

Materials/tngredients

Acre

$\underline{\text { Cost/Acre }}$

$\underline{\text { Cost/Acre }}$

Lorsban 4EC

$5 \mathrm{pts}$

$\$ 23.50$

Ground Application

(engine driven airblast)

500 gals $\quad \underline{38.00}$

Total per Application

$\$ \underline{\underline{61.50}}$

$\underline{\text { FALL SPRAY }}$

\begin{tabular}{|c|c|c|c|c|}
\hline \multirow[t]{5}{*}{ Spray Option \#11 } & $\underline{\text { Materials/Ingredients }}$ & $\begin{array}{l}\text { Amount } \\
\text { /Acre } \\
\end{array}$ & $\underline{\text { Cost/Acre }}$ & $\begin{array}{c}\text { Your } \\
\text { Cost/Acre } \\
\end{array}$ \\
\hline & Vendex 50WP & $2 \mathrm{lbs}$ & $\$ 32.70$ & \\
\hline & Microthiol (sulfur) & $15 \mathrm{lbs}$ & 11.55 & \\
\hline & $\begin{array}{l}\text { Ground Application } \\
\text { (PTO driven airblast) }\end{array}$ & 250 gals & $\underline{32.92}$ & \\
\hline & Total per Application & & $\$ 77.17$ & \\
\hline \multirow[t]{4}{*}{ Spray Option \#12 } & $\underline{\text { Materials/Ingredients }}$ & $\begin{array}{l}\text { Amount } \\
\text { /Acre } \\
\end{array}$ & $\underline{\text { Cost/Acre }}$ & $\begin{array}{c}\text { Your } \\
\text { Cost/Acre } \\
\end{array}$ \\
\hline & Vendex WP & $2 \mathrm{lbs}$ & $\$ 32.70$ & \\
\hline & $\begin{array}{l}\text { Ground Application } \\
\text { (PTO driven airblast) }\end{array}$ & $125 \mathrm{GPA}$ & $\underline{28.03}$ & \\
\hline & Total per Application & & $\$ \underline{\underline{60.73}}$ & \\
\hline \multirow[t]{4}{*}{ Spray Option \#13 } & $\underline{\text { Materials/Ingredients }}$ & $\begin{array}{l}\text { Amount } \\
\text { /Acre } \\
\end{array}$ & $\underline{\text { Cost/Acre }}$ & $\begin{array}{c}\text { Your } \\
\text { Cost/Acre } \\
\end{array}$ \\
\hline & Microthiol (sulfur) & $15 \mathrm{lbs}$ & $\$ 11.55$ & \\
\hline & Aerial Application & 15 GPA & $\underline{8.82}$ & \\
\hline & Total per Application & & $\$ \underline{\underline{20.37}}$ & \\
\hline
\end{tabular}

Table 2-A.--Herbicide options

Herbicide Option \#1

(Strip/band) $\underline{\text { Materials }}$

Solicam 80DF

Karmex WP

Roundup Ultra Max

Ground Application (1 time)

Total for 1 Application

\begin{tabular}{|c|c|c|}
\hline $\begin{array}{c}\text { Amount/ } \\
\text { Treated Acre }\end{array}$ & $\begin{array}{c}\text { Cost/ } \\
\text { Grove Acre }^{\mathrm{a}}\end{array}$ & $\begin{array}{r}\text { Your Cost/ } \\
\text { Grove Acre } \\
\end{array}$ \\
\hline $3 \mathrm{lbs}$ & $\$ 23.51$ & \\
\hline $4 \mathrm{lbs}$ & 8.52 & \\
\hline 2 qts & 8.02 & \\
\hline & 12.75 & \\
\hline
\end{tabular}

$\$ \underline{\underline{52.80}}$ 
Table 2-A.-Herbicide options (cont'd.)

\begin{tabular}{|c|c|c|c|c|}
\hline \multirow{6}{*}{$\begin{array}{l}\text { Herbicide Option \#2 } \\
\text { (Strip/band) }\end{array}$} & $\underline{\text { Materials }}$ & $\begin{array}{c}\text { Amount/ } \\
\text { Treated Acre } \\
\end{array}$ & $\begin{array}{c}\text { Cost/ } \\
\text { Grove Acre }^{\mathrm{a}}\end{array}$ & $\begin{array}{r}\text { Your Cost/ } \\
\text { Grove Acre } \\
\end{array}$ \\
\hline & Surflan A80 DF & $2 \mathrm{qts}$ & $\$ 22.48$ & \\
\hline & Simazine $4 \mathrm{~L}$ & $4 \mathrm{qts}$ & 7.56 & \\
\hline & Roundup Ultra Max & $2 \mathrm{qts}$ & 8.02 & \\
\hline & $\begin{array}{l}\text { Ground Application } \\
\text { (1 time) }\end{array}$ & & $\underline{12.75}$ & \\
\hline & Total for 1 Application & & $\$ \underline{\underline{50.81}}$ & \\
\hline \multirow{5}{*}{$\begin{array}{l}\text { Herbicide Option } \# 3 \\
\text { (Strip/band) }\end{array}$} & $\underline{\text { Materials }}$ & $\begin{array}{c}\text { Amount/ } \\
\text { Treated Acre } \\
\end{array}$ & $\begin{array}{c}\text { Cost/ } \\
\text { Grove Acre }^{\mathrm{a}}\end{array}$ & $\begin{array}{r}\text { Your Cost/ } \\
\text { Grove Acre } \\
\end{array}$ \\
\hline & Karmex WP & $4 \mathrm{lbs}$ & $\$ 8.52$ & \\
\hline & Roundup Ultra Max & 2 qts & 8.02 & \\
\hline & $\begin{array}{l}\text { Ground Application } \\
\quad(1 \text { time })\end{array}$ & & $\underline{12.75}$ & \\
\hline & Total for 1 Application & & $\$ 29.29$ & \\
\hline \multirow{6}{*}{$\begin{array}{l}\text { Herbicide Option \#4 } \\
\text { (Strip/band) }\end{array}$} & $\underline{\text { Materials }}$ & $\begin{array}{c}\text { Amount/ } \\
\text { Treated Acre } \\
\end{array}$ & $\begin{array}{c}\text { Cost/ } \\
\text { Grove Acre }^{\mathrm{a}}\end{array}$ & $\begin{array}{r}\text { Your Cost/ } \\
\text { Grove Acre } \\
\end{array}$ \\
\hline & Solicam 80DF & $4 \mathrm{lbs}$ & $\$ 23.51$ & \\
\hline & Simazine 4L & $4 \mathrm{qts}$ & 7.56 & \\
\hline & Roundup Ultra Max & $2 \mathrm{qts}$ & 8.02 & \\
\hline & $\begin{array}{l}\text { Ground Application } \\
\quad(1 \text { time })\end{array}$ & & $\underline{12.75}$ & \\
\hline & Total for 1 Application & & $\$ \underline{\underline{51.84}}$ & \\
\hline \multirow{4}{*}{$\begin{array}{l}\text { Herbicide Option \#5 } \\
\text { (Strip/band) }\end{array}$} & $\underline{\text { Materials }}$ & $\begin{array}{c}\text { Amount/ } \\
\text { Treated Acre } \\
\end{array}$ & $\begin{array}{c}\text { Cost/ } \\
\text { Grove Acre }^{\mathrm{a}}\end{array}$ & $\begin{array}{r}\text { Your Cost/ } \\
\text { Grove Acre } \\
\end{array}$ \\
\hline & Roundup Ultra Max & $2 \mathrm{qts}$ & $\$ 8.02$ & \\
\hline & $\begin{array}{l}\text { Ground Application } \\
\text { (1 time) }\end{array}$ & & $\underline{12.75}$ & \\
\hline & Total for 1 Application & & $\$ \underline{\underline{20.77}}$ & \\
\hline \multirow{5}{*}{$\begin{array}{l}\text { Herbicide Option \#6 } \\
\text { (Strip/band) }\end{array}$} & $\underline{\text { Materials }}$ & $\begin{array}{c}\text { Amount/ } \\
\text { Treated Acre } \\
\end{array}$ & $\begin{array}{c}\text { Cost/ } \\
\text { Grove Acre }^{\mathrm{a}}\end{array}$ & $\begin{array}{r}\text { Your Cost/ } \\
\text { Grove Acre } \\
\end{array}$ \\
\hline & Krovar I & $5 \mathrm{lbs}$ & $\$ 31.30$ & \\
\hline & Roundup Ultra Max & $2 \mathrm{qts}$ & 8.02 & \\
\hline & $\begin{array}{l}\text { Ground Application } \\
\text { (1 time) }\end{array}$ & & $\underline{12.75}$ & \\
\hline & Total for 1 Application & & $\$ \underline{\underline{52.07}}$ & \\
\hline
\end{tabular}

${ }^{a}$ With respect to herbicide materials, Amount Per Grove Acre does not equal Amount Per Treated Acre shown on the label. Only a strip or band is being treated. In this report, it is assumed that only one-half of a grove surface is being treated. 
Table 2-A.-Herbicide options (cont'd.)

\begin{tabular}{|c|c|c|c|c|}
\hline Herbicide Option \#7 & $\underline{\text { Materials }}$ & $\begin{array}{c}\text { Amount/ } \\
\text { Treated Acre } \\
\end{array}$ & $\begin{array}{c}\text { Cost/ } \\
\text { Grove Acre }^{\mathrm{a}}\end{array}$ & $\begin{array}{r}\text { Your Cost/ } \\
\text { Grove Acre } \\
\end{array}$ \\
\hline \multirow[t]{4}{*}{ (Strip/band) } & Roundup Ultra Max & $2 \mathrm{qts}$ & $\$ 8.02$ & \\
\hline & Princep (Caliber 90) & $4 \mathrm{lbs}$ & 7.24 & \\
\hline & $\begin{array}{l}\text { Ground Application } \\
\text { (1 time) }\end{array}$ & & $\underline{12.75}$ & \\
\hline & Total for 1 Application & & $\$ 28.01$ & \\
\hline \multirow{5}{*}{$\begin{array}{l}\text { Herbicide Option \#8 } \\
\text { (Strip/band) }\end{array}$} & $\underline{\text { Materials }}$ & $\begin{array}{c}\text { Amount/ } \\
\text { Treated Acre } \\
\end{array}$ & $\begin{array}{c}\text { Cost/ } \\
\text { Grove Acre }^{\mathrm{a}}\end{array}$ & $\begin{array}{r}\text { Your Cost/ } \\
\text { Grove Acre } \\
\end{array}$ \\
\hline & Direx 4L & $3 \mathrm{qts}$ & $\$ 6.84$ & \\
\hline & Solicam & $3 \mathrm{lbs}$ & 23.51 & \\
\hline & $\begin{array}{l}\text { Ground Application } \\
\text { (1 time) }\end{array}$ & & $\underline{12.75}$ & \\
\hline & Total for 1 Application & & $\$ \underline{\underline{43.10}}$ & \\
\hline \multirow{4}{*}{$\begin{array}{l}\text { Herbicide Option \#9 } \\
\text { (Chemical mow) }\end{array}$} & $\underline{\text { Materials }}$ & $\begin{array}{c}\text { Amount/ } \\
\text { Treated Acre } \\
\end{array}$ & $\begin{array}{c}\text { Cost/ } \\
\text { Grove Acre }^{\mathrm{a}}\end{array}$ & $\begin{array}{r}\text { Your Cost/ } \\
\text { Grove Acre } \\
\end{array}$ \\
\hline & Roundup Ultra Max & $1 \mathrm{pt}$ & $\$ 2.01$ & \\
\hline & $\begin{array}{l}\text { Ground Application } \\
\text { (1 time) }\end{array}$ & & 3.07 & \\
\hline & Total for 1 Application & & $\$ \underline{\underline{5.08}}$ & \\
\hline \multirow{4}{*}{$\begin{array}{l}\text { Herbicide Option \#10 } \\
\text { (Chemical mow) }\end{array}$} & $\underline{\text { Materials }}$ & $\begin{array}{c}\text { Amount/ } \\
\text { Treated Acre }\end{array}$ & $\begin{array}{c}\text { Cost/ } \\
\text { Grove Acre }^{\mathrm{a}} \\
\end{array}$ & $\begin{array}{r}\text { Your Cost/ } \\
\text { Grove Acre } \\
\end{array}$ \\
\hline & Roundup Ultra Max & $1.5 \mathrm{pts}$ & $\$ 3.02$ & \\
\hline & $\begin{array}{l}\text { Ground Application } \\
\quad(1 \text { time })\end{array}$ & & 3.07 & \\
\hline & Total for 1 Application & & $\$ \underline{\underline{6.09}}$ & \\
\hline \multirow{4}{*}{$\begin{array}{l}\text { Herbicide Option \#11 } \\
\text { (Spot treatment for } \\
\text { grass/brush regrowth } \\
\text { under trees) }\end{array}$} & $\underline{\text { Materials }}$ & $\begin{array}{c}\text { Amount/ } \\
\text { Treated Acre } \\
\end{array}$ & $\begin{array}{c}\text { Cost/ } \\
\text { Grove Acre }^{\mathrm{a}}\end{array}$ & \multirow[t]{4}{*}{$\begin{array}{r}\text { Your Cost } \\
\text { Grove Acre } \\
\end{array}$} \\
\hline & Roundup Ultra Max & $2 \mathrm{qts}$ & $\$ 8.02$ & \\
\hline & $\begin{array}{l}\text { Ground Application } \\
\quad(1 \text { time })\end{array}$ & & $\underline{4.56}$ & \\
\hline & Total for 1 Application & & $\$ \underline{\underline{12.58}}$ & \\
\hline
\end{tabular}


Table 3-A.--Dry fertilizer options

\begin{tabular}{|c|c|c|c|c|}
\hline Option \#1 & $\begin{array}{l}\text { Analysis/Material } \\
\text { Applied }\end{array}$ & $\begin{array}{l}\text { Amount } \\
\text { /Acre } \\
\end{array}$ & Cost/Acre & $\begin{array}{c}\text { Your } \\
\text { Cost/Acre } \\
\end{array}$ \\
\hline \multirow[t]{3}{*}{ (100 lbs N/Acre) } & $12-2-12-2.4 \mathrm{MgO}$ & $835 \mathrm{lbs}$ & $\$ 93.52$ & \\
\hline & Application & 3 times & 23.70 & \\
\hline & Total for 3 Applications & & $\$ \underline{\underline{117.22}}$ & \\
\hline \multirow[t]{4}{*}{ Option \#2 } & $\begin{array}{l}\text { Analysis/Material } \\
\text { Applied }\end{array}$ & $\begin{array}{l}\text { Amount } \\
\text { /Acre } \\
\end{array}$ & Cost/Acre & $\begin{array}{c}\text { Your } \\
\text { Cost/Acre } \\
\end{array}$ \\
\hline & $12-2-12-2.4 \mathrm{MgO}$ & $1040 \mathrm{lbs}$ & $\$ 116.48$ & \\
\hline & Application & 3 times & 23.70 & \\
\hline & Total for 3 Applications & & $\$ \underline{\underline{140.18}}$ & \\
\hline \multirow[t]{4}{*}{ Option \#3 } & $\begin{array}{l}\text { Analysis/Material } \\
\text { Applied }\end{array}$ & $\begin{array}{l}\text { Amount } \\
\text { /Acre } \\
\end{array}$ & $\underline{\text { Cost/Acre }}$ & $\begin{array}{c}\text { Your } \\
\text { Cost/Acre } \\
\end{array}$ \\
\hline & $12-2-12-2.4 \mathrm{MgO}$ & $1350 \mathrm{lbs}$ & $\$ 151.20$ & \\
\hline & Application & 3 times & 23.70 & \\
\hline & Total for 3 Applications & & $\$ \underline{\underline{174.90}}$ & \\
\hline \multirow{4}{*}{$\begin{array}{l}\text { Option \#4 } \\
\text { (180 lbs N/Acre) }\end{array}$} & $\begin{array}{l}\text { Analysis/Material } \\
\text { Applied }\end{array}$ & $\begin{array}{l}\text { Amount } \\
\text { /Acre } \\
\end{array}$ & $\underline{\text { Cost/Acre }}$ & $\begin{array}{c}\text { Your } \\
\text { Cost/Acre } \\
\end{array}$ \\
\hline & $15-2-15-2.4 \mathrm{MgO}$ & $1200 \mathrm{lbs}$ & $\$ 150.00$ & \\
\hline & Application & 3 times & 23.70 & \\
\hline & Total for 3 Applications & & $\$ \underline{\underline{173.70}}$ & \\
\hline \multirow{4}{*}{$\begin{array}{l}\text { Option } \# 5 \\
\text { (204 lbs N/Acre) }\end{array}$} & $\begin{array}{l}\text { Analysis/Material } \\
\text { Applied }\end{array}$ & $\begin{array}{l}\text { Amount } \\
\text { /Acre } \\
\end{array}$ & $\underline{\text { Cost/Acre }}$ & $\begin{array}{c}\text { Your } \\
\text { Cost/Acre } \\
\end{array}$ \\
\hline & $17-4-17-2.4 \mathrm{MgO}$ & $1200 \mathrm{lbs}$ & $\$ 157.20$ & \\
\hline & Application & 3 times & 23.70 & \\
\hline & Total for 2 Applications & & $\$ \underline{\underline{180.90}}$ & \\
\hline \multirow{4}{*}{$\begin{array}{l}\text { Option \#6 } \\
\text { (225 lbs N/Acre) }\end{array}$} & $\begin{array}{l}\text { Analysis/Material } \\
\text { Applied }\end{array}$ & $\begin{array}{l}\text { Amount } \\
\text { /Acre } \\
\end{array}$ & Cost/Acre & $\begin{array}{c}\text { Your } \\
\text { Cost/Acre } \\
\end{array}$ \\
\hline & $15-2-15-2.4 \mathrm{MgO}$ & $1500 \mathrm{lbs}$ & $\$ 187.50$ & \\
\hline & Application & 3 times & 23.70 & \\
\hline & Total for 3 Applications & & $\$ 211.20$ & \\
\hline
\end{tabular}


Table 4-A.--Liquid fertilizer (Double boom application)

\begin{tabular}{|c|c|c|c|c|}
\hline Option \#1 & $\begin{array}{l}\text { Analysis/Material } \\
\text { Applied }\end{array}$ & $\begin{array}{l}\text { Amount } \\
\text { /Acre } \\
\end{array}$ & Cost/Acre & $\begin{array}{c}\text { Your } \\
\text { Cost/Acre } \\
\end{array}$ \\
\hline \multirow{3}{*}{ (180 lbs N/Acre) } & $10-0-10$ & $1800 \mathrm{lbs}$ & $\$ 167.40$ & \\
\hline & Double Boom Application & 3 times & 37.65 & \\
\hline & Total for 3 Applications & & $\$ 205.05$ & \\
\hline \multirow{4}{*}{$\begin{array}{l}\text { Option \#2 } \\
\text { (180 lbs N/Acre) }\end{array}$} & $\begin{array}{l}\text { Analysis/Material } \\
\text { Applied }\end{array}$ & $\begin{array}{l}\text { Amount } \\
\text { /Acre }\end{array}$ & $\underline{\text { Cost/Acre }}$ & $\begin{array}{c}\text { Your } \\
\text { Cost/Acre } \\
\end{array}$ \\
\hline & $10-2-10$ & $1800 \mathrm{lbs}$ & $\$ 176.40$ & \\
\hline & Double Boom Application & 3 times & 37.65 & \\
\hline & Total for 3 Applications & & $\$ \underline{\underline{214.05}}$ & \\
\hline \multirow{6}{*}{$\begin{array}{l}\text { Option \#3 } \\
\text { (180 lbs N/Acre) }\end{array}$} & $\begin{array}{l}\text { Analysis/Material } \\
\text { Applied }\end{array}$ & $\begin{array}{l}\text { Amount } \\
\text { /Acre } \\
\end{array}$ & $\underline{\text { Cost/Acre }}$ & $\begin{array}{c}\text { Your } \\
\text { Cost/Acre } \\
\end{array}$ \\
\hline & $10-0-10$ & $1800 \mathrm{lbs}$ & $\$ 167.40$ & \\
\hline & Solicam 80DF & 3 lbs* & 23.51 & \\
\hline & Karmex WP & $4 \mathrm{lbs}^{*}$ & 8.52 & \\
\hline & Double Boom Application & 3 times & 37.65 & \\
\hline & Total for 3 Applications & & $\$ 237.08$ & \\
\hline
\end{tabular}

Table 5-A.--Nematicides options

Option \#1

Analysis/Material

Applied

Temik $15 \mathrm{G}$

Application

Total per Application
Amount /Acre $33 \mathrm{lbs}$

$\underline{\text { Cost/Acre }}$

Your

1 time

$\$ 116.16$

$\underline{11.34}$

$\$ \underline{\underline{127.50}}$

Table 6-A.--Soil amendment options

\begin{tabular}{|c|c|c|c|c|}
\hline \multirow{5}{*}{$\begin{array}{l}\text { Option \#1 } \\
\text { (Every } 3 \text { years) }\end{array}$} & $\begin{array}{l}\text { Analysis/Material } \\
\text { Applied }\end{array}$ & $\begin{array}{l}\text { Amount } \\
\text { /Acre }\end{array}$ & Cost/Acre & $\begin{array}{c}\text { Your } \\
\text { Cost/Acre } \\
\end{array}$ \\
\hline & Dolomite (Delivered) & 1 ton & $\$ 36.05$ & \\
\hline & Application & 1 time & 7.90 & \\
\hline & Total for 1 Application & & $\$ 43.95$ & \\
\hline & (Average $1 / 3$ Ton Applied/Yr) & & $\$ \underline{14.65}$ & \\
\hline \multirow{4}{*}{$\begin{array}{l}\text { Option \#2 } \\
\text { (Every year) }\end{array}$} & $\begin{array}{l}\text { Analysis/Material } \\
\text { Applied }\end{array}$ & $\begin{array}{l}\text { Amount } \\
\text { /Acre }\end{array}$ & Cost/Acre & $\begin{array}{c}\text { Your } \\
\text { Cost/Acre } \\
\end{array}$ \\
\hline & Dolomite (Delivered) & $1000 \mathrm{lbs}$ & $\$ 18.03$ & \\
\hline & Application & 1 time & 7.90 & \\
\hline & Total per Application & & $\$ 25.93$ & \\
\hline
\end{tabular}


Table 7-A.--Irrigation--annual cost per acre

$\underline{\text { DRIP }}$

\begin{tabular}{|c|c|c|c|c|}
\hline & $\underline{\text { Option \#1 }}$ & $\begin{array}{c}\text { Your } \\
\text { Cost/Acre } \\
\end{array}$ & $\underline{\text { Option \#2 }}$ & $\begin{array}{c}\text { Your } \\
\text { Cost/Acre }\end{array}$ \\
\hline Operating & $\begin{array}{l}\text { (Electric) } \\
\$ 62.10\end{array}$ & & $\begin{array}{c}\text { (Diesel) } \\
\$ 55.87\end{array}$ & \\
\hline Maintenance of System & 44.04 & & 43.82 & \\
\hline Total Cash Expenses & $\$ 106.14$ & & $\$ 99.69$ & \\
\hline Fixed Depreciation Expense & 42.35 & & 42.25 & \\
\hline Total Cash and Fixed & $\$ \underline{\underline{148.49}}$ & & $\$ \underline{\underline{144.91}}$ & \\
\hline
\end{tabular}

\section{MICROSPRINKLER}

\begin{tabular}{|c|c|c|c|c|}
\hline & $\underline{\text { Option \#3 }}$ & $\begin{array}{c}\text { Your } \\
\text { Cost/Acre } \\
\end{array}$ & $\underline{\text { Option \#4 }}$ & $\begin{array}{c}\text { Your } \\
\text { Cost/Acre }\end{array}$ \\
\hline Operating & $\begin{array}{l}\text { (Electric) } \\
\$ 70.60\end{array}$ & & $\begin{array}{c}\text { (Diesel) } \\
\$ 59.44^{*}\end{array}$ & \\
\hline Maintenance of System & 49.08 & & 50.17 & \\
\hline Total Cash Expenses & $\$ 119.68$ & & $\$ 109.61$ & \\
\hline Fixed Depreciation Expense & 52.94 & & $\underline{56.56}$ & \\
\hline Total Cash and Fixed & $\$ 172.62$ & & $\$ 166.17$ & \\
\hline
\end{tabular}
Expenses

DRAINAGE DITCH ANNUAL COSTS

\begin{tabular}{cc} 
Your \\
Option \#5 & Cost/Acre \\
\hline
\end{tabular}

Ditches/Canals Maintenance $(\$ 45.17 /$ acre $\div 3$ years)

$\$ 15.06$

Weed Control in Ditches/Canals

14.19

Water Control: In/Out of Ditches and Canals

$\underline{13.21}$

Total

$\$ \underline{\underline{42.36}}$

*Indicates higher cost for fuel; diesel or electric. 
Table 8-A.--A listing of 2005 custom rates reported by sixteen Indian River and South Florida citrus caretakers

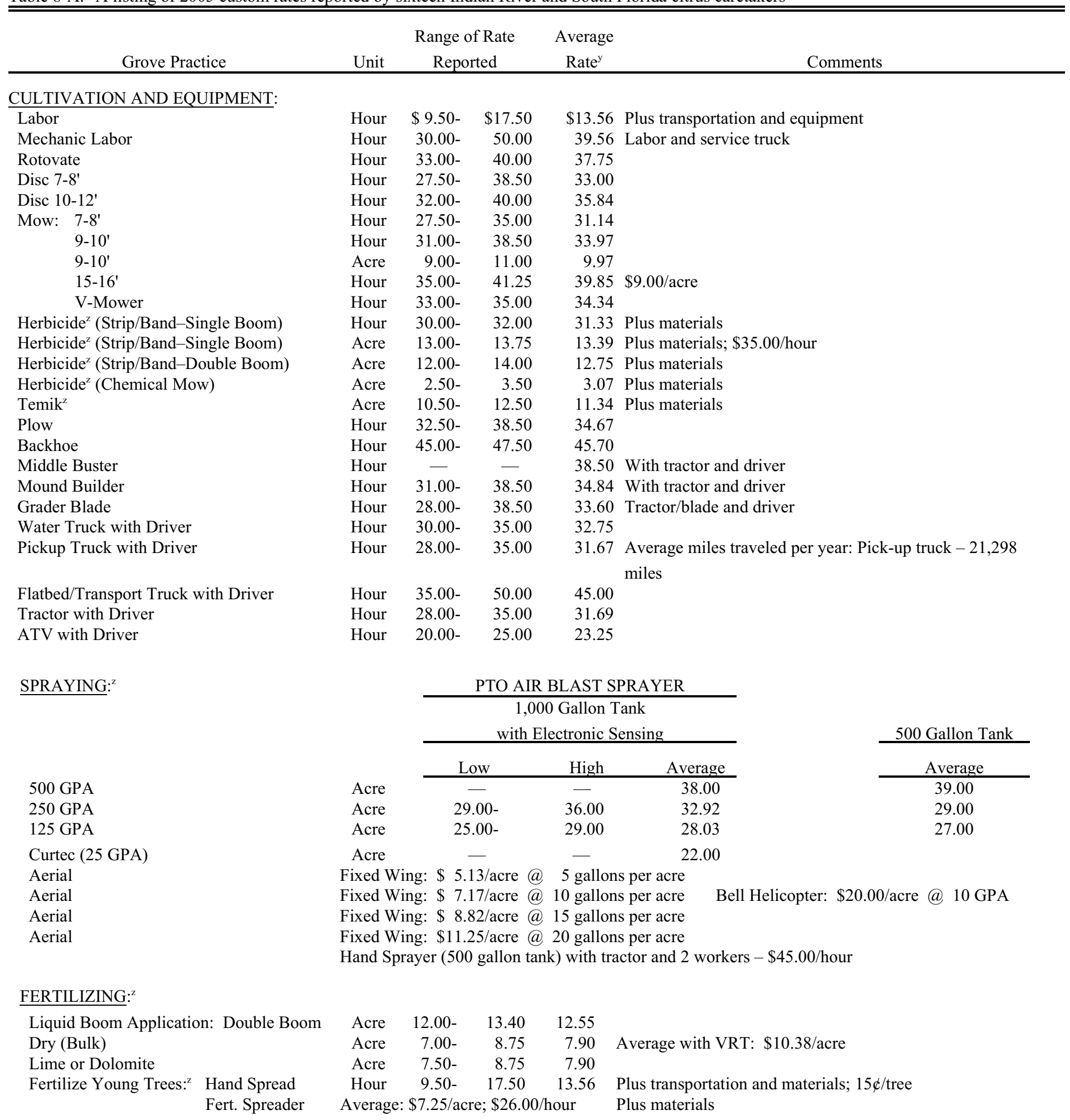


Table 8-A.--A listing of 2005 custom rates reported by sixteen Indian River and South Florida citrus caretakers (cont'd.)

\begin{tabular}{|c|c|c|c|c|c|}
\hline Grove Practice & Unit & \multicolumn{2}{|c|}{ Range of Rate } & Average & Comments \\
\hline \multicolumn{6}{|l|}{ IRRIGATION: } \\
\hline$\overline{\text { Ditch Mower }}$ & Hour & $\$ 32.00-$ & $\$ 44.50$ & $\$ 36.20$ & \\
\hline Water Furrow Disc & Hour & $30.00-$ & 38.50 & 34.67 & \\
\hline Water Furrow Cleaner & Hour & $35.00-$ & 38.50 & 36.34 & \\
\hline Water Furrow Shaper (Non-Laser Control) & Hour & - & - & 65.00 & \\
\hline Water Furrow Shaper (Laser Control) & Hour & - & - & 80.00 & \\
\hline Rotary Ditcher or Auger & Hour & $33.00-$ & 38.50 & 35.50 & \\
\hline Microsprinkler/Drip Irrigation Maintenance & Acre/Month & $3.50-$ & 4.75 & 4.25 & Check \& repair system; parts extra \\
\hline \multicolumn{6}{|l|}{ REMOVING TREES: } \\
\hline Front-end Loader & Hour & $\$ 50.00-$ & $\$ 65.00$ & $\$ 56.79$ & Avg. range $3-15$ trees per hour \\
\hline Tree Shearing (Cutting Tree at Ground Level) & Hour & $50.00-$ & 65.00 & 56.25 & Avg. range 5-20 trees per hour \\
\hline Prepare Site for Replanting & Tree & $\$ 0.25$ & $-\$ 1.00$ & & \\
\hline \multicolumn{6}{|l|}{ PRUNING: } \\
\hline \multicolumn{6}{|l|}{ Hedging: } \\
\hline Single Side (Tractor Mounted) & Hour & $\$-$ & $\$-$ & $\$ 55.00$ & \\
\hline Double Side (Tractor Pulled) & Hour & - & - & 65.00 & \\
\hline Double Side (Self Propelled) & Hour & $250.00-$ & 265.00 & 257.50 & $\begin{array}{l}8 \text { to } 20 \mathrm{~A} / \mathrm{H} \text { depending on wood size; } \$ 14 / \mathrm{A} \text { annual } \\
\text { cut }\end{array}$ \\
\hline Double Side Rotary (Self Propelled) ${ }^{\mathrm{x}}$ & Hour & - & - & 185.00 & 5 to $15 \mathrm{~A} / \mathrm{H}$ bed tops only; add $25 \%$ for furrows only \\
\hline \multicolumn{6}{|l|}{ Topping: } \\
\hline Double Sided Topper (Self Propelled) & Hour & $265.00-$ & 285.00 & 275.00 & $\begin{array}{l}\text { Avg. } 8-15 \text { ac depending on wood size type of } \\
\text { cut; } \$ 30 / \text { acre }\end{array}$ \\
\hline Topping Self Propelled & Hour & - & - & 150.00 & \\
\hline Limb Lifter/Tree Skirt Trimmer & Acre & - & - & 14.00 & 3 to 5 acres/hour \\
\hline Limb Lifter/Tree Skirt Trimmer (Double Sided & Hour & - & - & 120.00 & 6 to 20 acres/hour \\
\hline \multicolumn{6}{|l|}{ Rotary) } \\
\hline \multicolumn{6}{|l|}{ Removing Brush: } \\
\hline Haul Brush out of Grove (Front-End Loader) & Hour & $55.00-$ & 65.00 & 59.25 & \\
\hline Mow/Chop Brush & Hour & $32.00-$ & 40.00 & 34.60 & \\
\hline \multicolumn{6}{|l|}{ OTHER CUSTOM RATES: } \\
\hline Install Tree Wraps & \multicolumn{5}{|c|}{$15 \phi-50 \notin /$ tree depending on type of wrap and number of trees; Annual maintenance cost: } \\
\hline Plant Trees (Solid Set) & Tree & $\$ 0.90-$ & $\$ 1.75$ & $\$ 1.32$ & Varies as to density \\
\hline Plant Trees (Resets) & Tree & $2.00-$ & 2.50 & 2.17 & Varies as to the number of resets \\
\hline Travel/Setup Charge & Hour & - & - & 22.62 & \\
\hline \multicolumn{6}{|l|}{ Grove Management Charge/Month: } \\
\hline Supervising Grove Care Operations & Acre & $3.00-$ & 7.50 & 5.15 & In addition to caretaking charges \\
\hline Handling Fruit Marketing & \multirow{2}{*}{\multicolumn{5}{|c|}{$\begin{array}{l}\$ 0.10-\$ 0.25 / \text { box }- \text { For Supervising and Marketing fruit } \\
5 \% \text { to } 15 \% \text { of materials cost }\end{array}$}} \\
\hline Supervising/Handling Chemicals/Fertilizer & & & & & \\
\hline $\begin{array}{l}\text { Charge for personnel to oversee harvesting } \\
\text { operations and coordinate harvest in different } \\
\text { blocks/groves and keeping of harvesting labor } \\
\text { compliance records. }\end{array}$ & Box & $\$ 0.05-$ & $\$ 0.25$ & $\$ 0.15$ & \\
\hline Consulting & Hour & $\$ 125.00-$ & $\$ 200.00$ & $\$ 150.00$ & Horticultural Evaluation and/or Financial \\
\hline & & & & & Analysis/prospectus. \\
\hline Total Reported Acreage Provided Grove Service to: & Acre & $1,000-$ & 14,000 & 4,870 & Total acres reporting: 48,700 \\
\hline
\end{tabular}

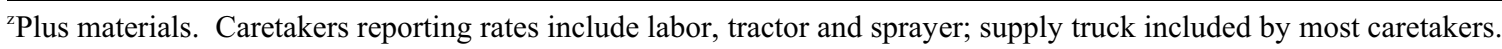

${ }^{\mathrm{y}}$ Calculated by dividing the total number of caretakers reporting a grove practice rate into the sum reported. Unless otherwise stated, labor included with all charges.

${ }^{\mathrm{x}}$ Low acres is for 2 years regrowth hedging; high acres is for annual maintenance hedging. 
Table 9-A.--2005 summary of average chemical price estimates

\begin{tabular}{|c|c|c|c|c|}
\hline \multicolumn{2}{|l|}{ Item } & Unit & $\begin{array}{c}\text { Average } \\
\text { Price }\end{array}$ & $\begin{array}{c}\text { Your Price } \\
(2005)\end{array}$ \\
\hline \multirow[t]{16}{*}{ Fungicides: } & Abound EC & gal. & 218.12 & \\
\hline & Aliette 80WP & lb. & 11.59 & \\
\hline & Basic Copper Sulfate & lb. & 1.40 & \\
\hline & Copper (Kocide 101) & lb. & 1.80 & \\
\hline & Copper (Kocide 2000) & lb. & 2.33 & \\
\hline & Copper (Champ II Flowable) & gal. & 22.55 & \\
\hline & Cuprofix Disperss & lb. & 1.75 & \\
\hline & $\mathrm{Nu}-\mathrm{Cop} 50 \mathrm{DF}$ & lb. & 1.88 & \\
\hline & Enable & gal. & 57.55 & \\
\hline & Gem 25 & 40 ozs. & 120.59 & \\
\hline & Headline EC & gal. & 206.13 & \\
\hline & Oil - 435 or 455 & gal. & 2.21 & \\
\hline & Oil - 470 (Bio-lever) & gal. & 2.46 & \\
\hline & Ridomil Gold EC & gal. & 649.15 & \\
\hline & Safe-T-Oil & gal. & 3.15 & \\
\hline & Topsin & lb. & 14.08 & \\
\hline \multicolumn{5}{|c|}{ Insecticides/Nematicides: } \\
\hline & $\overline{\text { Admire } 2 \mathrm{~F}}$ & gal. & 520.28 & \\
\hline & Agri-Mek (0.15EC) & gal. & 563.52 & \\
\hline & Award Fire Ant Bait & $\mathrm{lb}$. & 9.01 & \\
\hline & Bio-Vector & gal. & 412.50 & \\
\hline & Carbaryl 4L & gal. & 27.25 & \\
\hline & Carbaryl 80S & lb. & 4.47 & \\
\hline & Chlorpyrifos $4 \mathrm{E}$ & gal. & 57.26 & \\
\hline & Danitol & gal. & 147.58 & \\
\hline & Guthion 2L & gal. & 32.48 & \\
\hline & Guthion 50WP & lb. & 10.07 & \\
\hline & Imidan 70W (Diaprepes) & lb. & 8.25 & \\
\hline & Lorsban 4EC & gal. & 34.15 & \\
\hline & Lorsban 15G & lb. & 1.72 & \\
\hline & Malathion 5 EC & gal. & 25.18 & \\
\hline & Micromite $80 \mathrm{WG}$ & gal. & 87.95 & \\
\hline & Microthiol & lb. & 0.70 & \\
\hline & Nexter 75WP & lb. & 89.56 & \\
\hline & Provado 1.6 F (nursery) & gal. & 417.75 & \\
\hline & Sevin $80 \mathrm{~S}$ & lb. & 5.17 & \\
\hline & Sevin XLR & gal. & 30.96 & \\
\hline & Spintor $2 \mathrm{~S} \mathrm{C}$ & gal. & 492.50 & \\
\hline & Sulphur $6 \mathrm{~F}$ & gal. & 4.00 & \\
\hline & Temik 15G & lb. & 3.20 & \\
\hline & Vendex $50 \mathrm{~W}$ & lb. & 14.86 & \\
\hline & Vydate & gal. & 56.28 & \\
\hline
\end{tabular}


Table 9-A.--2005 summary of average chemical price estimates (cont'd.)

\begin{tabular}{|c|c|c|c|c|}
\hline \multicolumn{2}{|l|}{ Item } & \multirow{2}{*}{$\frac{\text { Unit }}{\text { gal. }}$} & \multirow{2}{*}{$\begin{array}{c}\begin{array}{c}\text { Average } \\
\text { Price }\end{array} \\
48.39\end{array}$} & \multirow[t]{2}{*}{$\begin{array}{c}\text { Your Price } \\
(2005) \\
\end{array}$} \\
\hline Herbicides: & Aqua Master & & & \\
\hline & Diuron 4L & gal. & 16.04 & \\
\hline & Direx 4L & gal. & 16.50 & \\
\hline & Direx $80 \mathrm{DF}$ & lb. & 3.87 & \\
\hline & Fusilade DX 2E & gal. & 131.14 & \\
\hline \multicolumn{5}{|c|}{ Glyphosate: } \\
\hline & Glyphomax Plus & gal. & 18.22 & \\
\hline & Roundup (Original) & gal. & 23.60 & \\
\hline & Roundup - Ultra Max & gal. & 29.12 & \\
\hline & Roundup Weather Max & gal. & 50.16 & \\
\hline & Roundup Original Max & gal. & 43.50 & \\
\hline & Touchdown & gal. & 37.05 & \\
\hline & Gramoxone E (Paraquat) & gal. & 37.53 & \\
\hline & Hyvar X 80 WP & lb. & 18.93 & \\
\hline & Karmex $80 \mathrm{DF}$ & lb. & 3.87 & \\
\hline & Krovar I & lb. & 11.38 & \\
\hline & Landmaster II & gal. & 18.66 & \\
\hline & Mandate 2E & gal. & 166.09 & \\
\hline & Pendimax & gal. & 24.37 & \\
\hline & Poast Plus 1.0 EC & gal. & 52.50 & \\
\hline & Princep (Caliber 90) & lb. & 3.29 & \\
\hline & Princep 4L & gal. & 14.51 & \\
\hline & Prowl & gal. & 22.12 & \\
\hline & Simazine $90 \mathrm{DF}$ & lb. & 2.80 & \\
\hline & Simazine 4L & gal. & 13.66 & \\
\hline & Solicam $80 \mathrm{DF}$ & lb. & 14.24 & \\
\hline & Simtrol & & 19.00 & \\
\hline & Surflan & gal. & 81.64 & \\
\hline \multicolumn{5}{|c|}{ Growth Regulators: } \\
\hline & Citrus Fix & gal. & 494.00 & \\
\hline & Pro-Gibb 3.91\% & 20 oz. bottle & 33.16 & \\
\hline & Tree-Hold & gal. & 79.17 & \\
\hline \multicolumn{5}{|c|}{ Other Spray Materials: } \\
\hline & Borates $(15 \%)$ & lb. & 0.70 & \\
\hline & Manganese (32\%) & lb. & 0.32 & \\
\hline & Zinc $(78 \%)$ & lb. & 0.83 & \\
\hline & Adjuvant (Surfactant) & gal. & 23.59 & \\
\hline
\end{tabular}

SOURCE: Ronald P. Muraro, Extension Farm Management Economist, University of Florida, IFAS, CREC, Lake Alfred, Florida, August 2005. 
Table 10-A.--2005 summary of average fertilizer price estimates

\begin{tabular}{|c|c|c|c|}
\hline Item & Unit & $\begin{array}{c}\text { Average } \\
\text { Price } \\
\end{array}$ & $\begin{array}{c}\text { Your Price } \\
(2005) \\
\end{array}$ \\
\hline \multicolumn{4}{|l|}{ FERTILIZER (FOB Price @ Plant) } \\
\hline & & $\$$ & \\
\hline \multicolumn{4}{|l|}{$\underline{\text { Dry Mix (Bulk) }}$} \\
\hline $17-0-17-3_{\mathrm{Mg}}$ & ton & 238.82 & \\
\hline $17-4-17-2.4_{\mathrm{Mg}}$ & ton & 243.35 & \\
\hline $16-0-16$ & ton & 218.35 & \\
\hline $16-0-16-4_{\mathrm{Mg}}$ & ton & 239.49 & \\
\hline $16-2-16-3_{\mathrm{Mg}}$ & ton & 240.45 & \\
\hline $15-2-15-2.4_{\mathrm{Mg}}$ & ton & 224.47 & \\
\hline $12-2-12-2.4_{\mathrm{Mg}}$ & ton & 201.02 & \\
\hline $8-8-8 \mathrm{w} / \mathrm{minors} *$ & ton & 182.90 & \\
\hline $8-4-8 \mathrm{w} /$ minors* & ton & 170.29 & \\
\hline $8-2-8 \mathrm{w} / \mathrm{minors} *$ & ton & 162.12 & \\
\hline 6-6-6 $\mathrm{w} /$ minors* & ton & 159.99 & \\
\hline \multicolumn{4}{|l|}{$\underline{\text { Liquid Mix (Bulk) }}$} \\
\hline $8-2-8$ & ton & 151.53 & \\
\hline $8-4-8$ & ton & 159.73 & \\
\hline $9-3-9$ & ton & 166.33 & \\
\hline $9-4-9$ & ton & 172.47 & \\
\hline $10-0-10$ & ton & 166.62 & \\
\hline $10-2-10$ & ton & 176.25 & \\
\hline $12-0-6$ & ton & 166.89 & \\
\hline $12-3-6$ & ton & 180.25 & \\
\hline 7-0-0-6 (Magnesium Nitrate) & ton & 218.00 & \\
\hline
\end{tabular}

*With organic nitrogen, the price averaged $25 \%$ higher. 
Table 10-A.--2005 summary of average fertilizer price estimates (cont'd.)

\begin{tabular}{|c|c|c|c|}
\hline Item & Unit & $\begin{array}{c}\text { Average } \\
\text { Price } \\
\end{array}$ & $\begin{array}{c}\text { Your Price } \\
(2005) \\
\end{array}$ \\
\hline \multicolumn{4}{|l|}{$\underline{\text { Other Fertilizer Materials (Bulk) }}$} \\
\hline Ammonium Nitrate (21\% N Liquid) & ton & 179.88 & \\
\hline Ammonium Nitrate (33.5\% N Dry) & ton & 259.38 & \\
\hline Ammonium Sulfate $(21 \% \mathrm{~N})$ & ton & 152.94 & \\
\hline Calcium Nitrate $(19 \% \mathrm{Ca}, 15.5 \% \mathrm{~N})$ & ton & 288.13 & \\
\hline Dolomite (at mine-- $49 \% \mathrm{CaCO}_{3}, 36 \% \mathrm{MgCO}_{3}$ ) & ton & 19.75 & \\
\hline Muriate of Potash $\left(60 \% \mathrm{~K}_{2} \mathrm{O}\right)$ & ton & 242.29 & \\
\hline Potassium Nitrate $\left(14 \% \mathrm{~N} ; 46 \% \mathrm{~K}_{2} \mathrm{O}\right)$ & ton & 453.57 & \\
\hline Sul-Po-Mag (SPM--21.9\% $\left.\mathrm{K}_{2} \mathrm{O}\right)$ & ton & 202.43 & \\
\hline Super Phosphate $\left(20 \% \mathrm{P}_{2} \mathrm{O}_{5}\right)$ & ton & 214.25 & \\
\hline Triple Superphosphate $\left(48 \% \mathrm{P}_{2} \mathrm{O}_{5}\right)$ & ton & 242.92 & \\
\hline Average Delivery Cost & ton & 14.32 & \\
\hline \multicolumn{4}{|l|}{$\underline{\text { Foliar Macronutrients }}$} \\
\hline Phos Might 0-22-20 & gal. & 24.29 & \\
\hline Nutriphite Magnum 2-40-16 & gal. & 35.00 & \\
\hline MKP (0-52-34) (Mono-Potassium Phosphate) & lb. & 0.80 & \\
\hline RSA ActaPhos 0-28-25 & gal. & 18.00 & \\
\hline Peter's 20-20-20 Foliar & lb. & 0.54 & \\
\hline $\mathrm{MZF}$ & gal. & 6.53 & \\
\hline \multicolumn{4}{|l|}{$\underline{\text { Slow Release Nitrogen (SRN) }}$} \\
\hline \multicolumn{4}{|l|}{$\underline{\text { CitriBlen }}$} \\
\hline $15-3-19$ & ton & 245.15 & \\
\hline $17-5-12$ & ton & 237.50 & \\
\hline $18-6-11$ & ton & 243.80 & \\
\hline Sulfur Coated Urea (SCU) & ton & 586.80 & \\
\hline Agriform 20-10-5 (500 tablets/box) & box & 40.00 & \\
\hline
\end{tabular}

SOURCE: Ronald P. Muraro, Extension Farm Management Economist, University of Florida, IFAS, CREC, Lake Alfred, Florida, August 2005. 
Table 11-A.--Cost for establishing, planting and maintaining a citrus grove through four years of age, South Florida flatwoods area

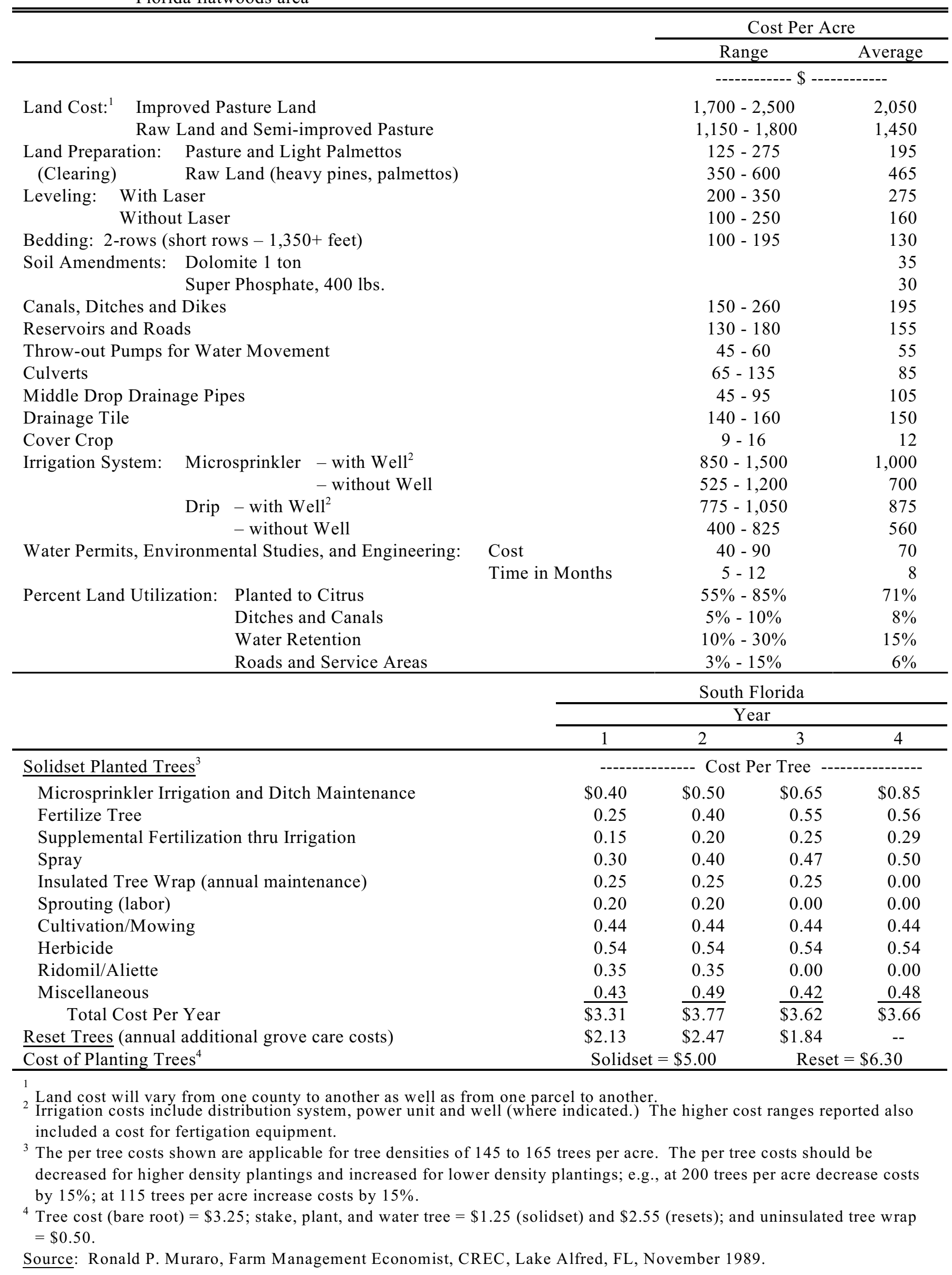


Table 12-A.--Estimated cost of planting and maintaining a reset citrus tree through three years of age, Southwest Florida area, August 2005

\begin{tabular}{|c|c|c|c|c|c|}
\hline & \multicolumn{5}{|c|}{ Resets/Replacement Trees Per Acre } \\
\hline & $1-2$ & $3-5$ & $6-10$ & $11-25$ & $26+$ \\
\hline & \multicolumn{5}{|c|}{ 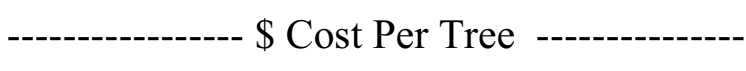 } \\
\hline Tree Removal & 6.67 & 5.34 & 4.45 & 3.56 & 2.67 \\
\hline \multicolumn{6}{|l|}{$\underline{\text { Plant ResetTree }}$} \\
\hline Tree Cost (Container Tree) & 4.50 & 4.50 & 4.35 & 4.35 & 4.35 \\
\hline Plant Tree and First Watering (Custom Charge) & 2.93 & 2.55 & $\underline{2.17}$ & 1.84 & 1.57 \\
\hline Total Planting Costs & 7.43 & 7.05 & 6.52 & 6.19 & 5.92 \\
\hline
\end{tabular}

$\underline{\text { Site Preparation }}^{\mathrm{a}}$

Rotovate

$\begin{array}{lllll}2.65 & 2.31 & 1.96 & 1.67 & 1.42 \\ \underline{3.00} & \underline{2.61} & \underline{2.22} & \underline{1.89} & \underline{1.60} \\ 5.65 & 4.92 & 4.18 & 3.56 & 3.02\end{array}$

Total Planting and Site Preparation Costs

$\begin{array}{lllll}13.08 & 11.97 & 10.70 & 9.75 & 8.94\end{array}$

\begin{tabular}{|c|c|c|c|c|c|c|}
\hline$\underline{\text { Supplemental Maintenance }}$ & Year \#1 & 4.13 & 3.82 & 3.59 & 3.39 & 3.19 \\
\hline (Trees 1-3 years old) & Year \#2 & 3.79 & 3.39 & 2.96 & 2.59 & 2.27 \\
\hline (Fertilizer, Tree Wraps, Sprout, etc.) & Year \#3 & $\underline{3.07}$ & $\underline{2.73}$ & $\underline{2.34}$ & 2.01 & $\underline{1.73}$ \\
\hline \multicolumn{2}{|c|}{ Total Supplemental Maintenance Costs } & 10.99 & 9.94 & 8.89 & 7.99 & 7.19 \\
\hline \multicolumn{2}{|l|}{ Summary of Tree Replacement Costs } & 1 & 3 & 6 & 6 & 6 \\
\hline \multicolumn{2}{|l|}{ Tree Removal Costs } & 6.67 & 5.34 & 4.45 & 3.56 & 2.67 \\
\hline \multicolumn{2}{|l|}{ Planting and Tree Removal Costs } & 13.08 & 11.97 & 10.70 & 9.75 & 8.94 \\
\hline \multicolumn{2}{|c|}{ Supplemental Maintenance Costs (Years 1 thru 3) } & $\underline{10.99}$ & $\underline{9.93}$ & $\underline{8.90}$ & $\underline{7.99}$ & $\underline{7.19}$ \\
\hline \multicolumn{2}{|l|}{ Total Three-Year Cumulative Costs } & $\underline{\underline{30.74}}$ & $\underline{\underline{27.24}}$ & $\underline{\underline{24.05}}$ & $\underline{21.30}$ & $\underline{18.80}$ \\
\hline
\end{tabular}

${ }^{\text {a Site }}$ preparation for bedded citrus grove. Fumigate planting site would cost approximately $\$ 2.50$ per tree.

Source: Ronald P. Muraro, Farm Management Economist, CREC, Lake Alfred, FL, August 2005. 
Table 13-A.--A listing of estimated comparative Indian River citrus production costs per acre for grapefruit, 2004-2005

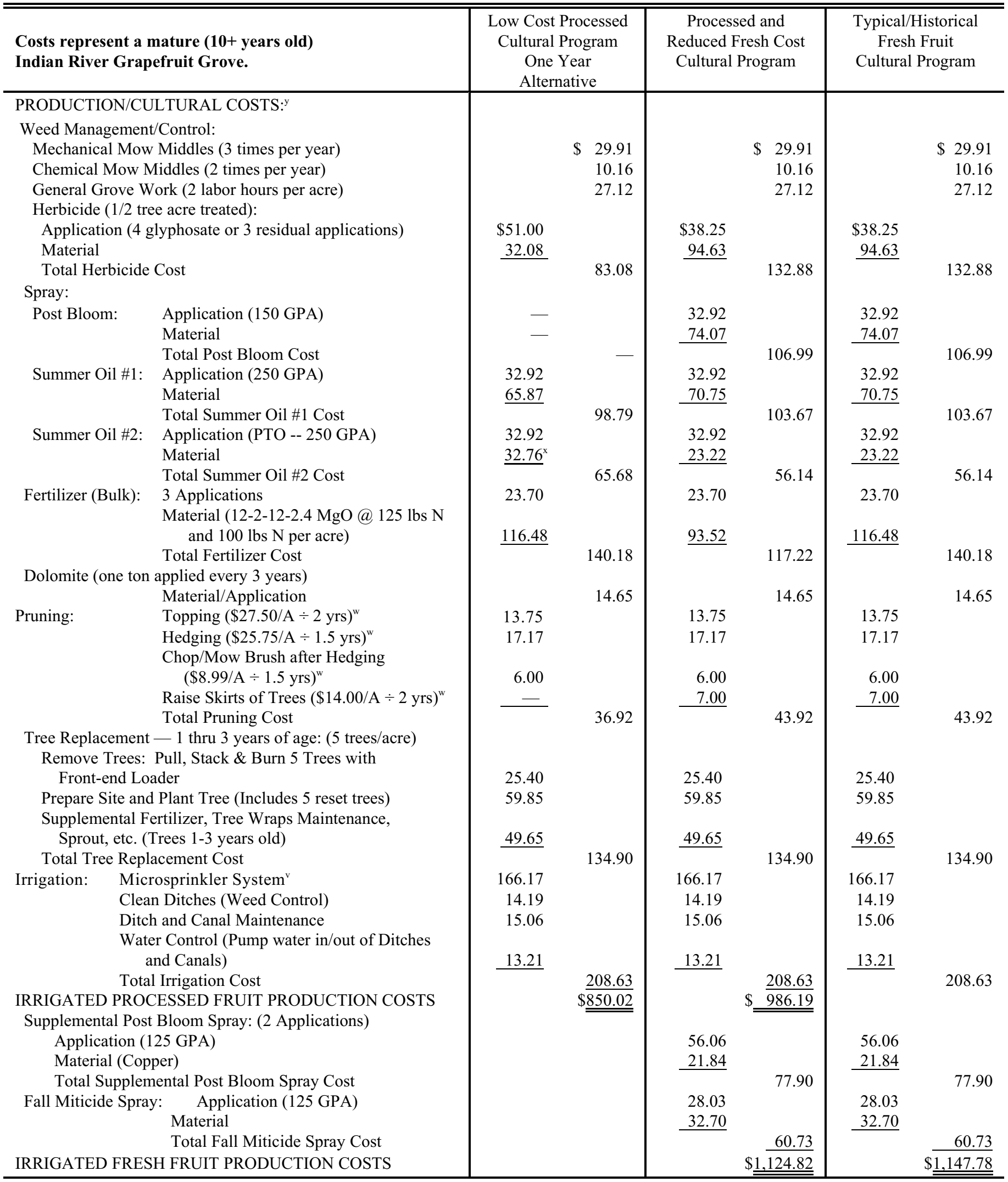

${ }^{z}$ The listed estimated comparative costs are for the example grove situation described in the Economic Information Report Series entitled:

"Budgeting Costs and Returns for Indian River Citrus Production" and may not represent your particular grove situation in Indian River. 
Table 14-A.-- Estimated average picking, roadsiding and hauling charges for Florida citrus, 2004-05

\begin{tabular}{|c|c|c|c|c|c|c|}
\hline & \multicolumn{3}{|c|}{ Fresh Fruit } & \multicolumn{3}{|c|}{ Processed Fruit } \\
\hline & & Range & Average & & Range & Average \\
\hline & & \$/Box & $\$ /$ Box & & \$/Box & $\$ /$ Box \\
\hline \multicolumn{7}{|l|}{ Picking Charges: } \\
\hline Early and Mid-Season Oranges & 0.70 & $-\quad 1.75$ & 0.954 & 0.65 & $-\quad 1.05$ & 0.829 \\
\hline Valencia Oranges & 0.70 & $-\quad 1.25$ & 0.938 & 0.65 & $-\quad 1.25$ & 0.870 \\
\hline Pink/Red Grapefruit & 0.60 & $-\quad 1.25$ & 0.739 & 0.55 & $-\quad 1.25$ & 0.669 \\
\hline White/Marsh Grapefruit & 0.60 & $-\quad 1.25$ & 0.744 & 0.55 & $-\quad 1.25$ & 0.667 \\
\hline Temples/Tangelos & 0.85 & -1.65 & 1.163 & 0.80 & $-\quad 1.50$ & 1.043 \\
\hline Tangerines & 1.25 & $-\quad 2.00$ & 1.529 & 1.00 & $-\quad 1.70$ & 1.204 \\
\hline \multirow[t]{4}{*}{ Add for Spot Picking } & 0.10 & $-\quad 0.50$ & 0.314 & & - & - \\
\hline & \multicolumn{3}{|c|}{ Fresh Fruit } & \multicolumn{3}{|c|}{ Processed Fruit } \\
\hline & \multicolumn{2}{|c|}{ Range } & Average & \multicolumn{2}{|r|}{ Range } & Average \\
\hline & \multicolumn{2}{|c|}{$\$ /$ Box } & $\$ /$ Box & \multicolumn{2}{|r|}{$\$ /$ Box } & \$/Box \\
\hline \multicolumn{7}{|l|}{ Roadsiding Charges: } \\
\hline Early and Mid-Season Oranges & 0.60 & $-\quad 1.15$ & 0.895 & 0.65 & $-\quad 1.17$ & 0.817 \\
\hline Valencia Oranges & 0.67 & $-\quad 1.12$ & 0.899 & 0.65 & $-\quad 1.17$ & 0.836 \\
\hline Pink/Red Grapefruit & 0.65 & $-\quad 1.03$ & 0.840 & 0.65 & $-\quad 1.20$ & 0.796 \\
\hline White/Marsh Grapefruit & 0.65 & $-\quad 1.03$ & 0.854 & 0.65 & $-\quad 1.20$ & 0.789 \\
\hline Temples/Tangelos & 0.70 & $-\quad 1.35$ & 1.003 & 0.75 & $-\quad 1.23$ & 0.890 \\
\hline \multirow[t]{4}{*}{ Tangerines } & 0.75 & $-\quad 1.35$ & 1.095 & 0.85 & $-\quad 1.70$ & 1.054 \\
\hline & \multicolumn{3}{|c|}{ Fresh Fruit } & \multicolumn{3}{|c|}{ Processed Fruit } \\
\hline & \multicolumn{3}{|c|}{ All Varieties } & \multicolumn{3}{|c|}{ All Varieties } \\
\hline & \multicolumn{3}{|c|}{$\$ /$ Box } & \multicolumn{3}{|c|}{ \$/Box } \\
\hline \multicolumn{7}{|l|}{ Hauling Charges: } \\
\hline 0 - 30 miles & \multicolumn{3}{|c|}{0.417} & \multicolumn{3}{|c|}{0.393} \\
\hline $31-50$ miles & \multicolumn{3}{|c|}{0.512} & \multicolumn{3}{|c|}{0.464} \\
\hline $51-80$ miles & \multicolumn{3}{|c|}{0.573} & \multicolumn{3}{|c|}{0.515} \\
\hline $81-100$ miles & \multicolumn{3}{|c|}{0.640} & \multicolumn{3}{|c|}{0.632} \\
\hline $100+$ miles & \multicolumn{3}{|c|}{0.746} & \multicolumn{3}{|c|}{0.728} \\
\hline
\end{tabular}


Table 15-A.--Estimated Average Packing Charges for Florida Citrus, 2004-05

\begin{tabular}{|c|c|c|c|c|c|}
\hline & $\begin{array}{l}\text { Domestic } \\
\text { Grapefruit }\end{array}$ & $\begin{array}{c}\text { Export } \\
\text { Grapefruit }\end{array}$ & Oranges & $\begin{array}{l}\text { Temples/ } \\
\text { Tangelos }\end{array}$ & Tangerines \\
\hline \multirow[b]{2}{*}{ Total Packing Charge $^{\mathrm{b}}$} & \multicolumn{5}{|c|}{ 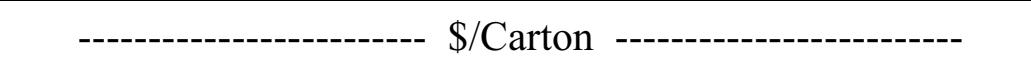 } \\
\hline & 3.835 & 4.245 & 4.192 & 4.495 & 5.056 \\
\hline & \multicolumn{5}{|c|}{$\begin{array}{l} \\
\end{array}$} \\
\hline Drenching Charge & 0.178 & 0.178 & 0.186 & 0.186 & 0.186 \\
\hline $\begin{array}{l}\text { Packinghouse Elimination } \\
\text { Charges }\end{array}$ & 0.594 & 0.594 & 0.571 & 0.571 & 0.571 \\
\hline $\begin{array}{l}\text { Hauling Charges for } \\
\text { Eliminations }\end{array}$ & 0.425 & 0.425 & 0.410 & 0.410 & 0.410 \\
\hline
\end{tabular}

${ }^{\text {aP }}$ acking charges represents a total of nine citrus packinghouses from both the Indian River and Interior production regions.

${ }^{\mathrm{b}}$ Total Packing Charge includes the following items:

1. Materials including mesh/plastic bags, labels/Price Lookup Codes (PLUs), etc.

2. Includes supervisor/foreman labor, grading, palletizing, shipping and general labor. Includes payroll taxes, workers' compensation, ground insurance, etc.

3. Other direct packing costs include: fruit treating; power, lights and water; repairs maintenance; miscellaneous supplies; etc.

4. Indirect packing costs include such items as: insurance-fire and casualty; taxes and licenses; depreciation and rent.

5. General and Administrative (G\&A) costs include: office personnel (payroll taxes, w/comp); packinghouse and general manager; office supplies; telephone; etc.

6. Selling Expenses which include sales salaries, travel, telephone and telegraph and brokerage fees.

7. Special assessments include such items as: advertising taxes; inspection fees; a Florida Citrus Packers tax; and a Citrus Administrative Committee (CAC) tax.

SOURCE: Ronald P. Muraro, University of Florida-IFAS, Citrus Research and Education Center, Lake Alfred, FL, September 2005. 
Table 16-A.--Historic prices ${ }^{\text {a }}$ for selected citrus varieties

\begin{tabular}{|c|c|c|c|c|c|c|c|}
\hline \multirow[b]{3}{*}{ Crop year } & \multicolumn{7}{|c|}{ Variety } \\
\hline & \multirow{2}{*}{$\begin{array}{c}\text { Early }{ }^{\mathrm{b}} \text { and } \\
\text { mid }^{\mathrm{c}} \text {-season } \\
\text { oranges }\end{array}$} & \multirow[b]{2}{*}{$\begin{array}{c}\text { Late season } \\
\text { oranges }^{\mathrm{d}}\end{array}$} & \multirow[b]{2}{*}{$\begin{array}{l}\text { Temple } \\
\text { oranges }\end{array}$} & \multirow[b]{2}{*}{$\begin{array}{c}\text { All } \\
\text { Tangerines } \\
\end{array}$} & \multirow[b]{2}{*}{ Tangelos } & \multicolumn{2}{|c|}{ Seedless grapefruit ${ }^{\mathrm{e}}$} \\
\hline & & & & & & (white) & (colored) \\
\hline $1961-62$ & $\$ 1.93$ & $\$ 1.81$ & $\$ 2.17$ & $\$ 2.04$ & $\$ 3.36$ & $\$ 0.68$ & $\$ 0.86$ \\
\hline $1962-63$ & 2.17 & 3.50 & 3.09 & 3.02 & 4.66 & 1.29 & 1.81 \\
\hline $1963-64$ & 4.43 & 4.45 & 4.45 & 3.18 & 4.83 & 2.24 & 2.54 \\
\hline $1964-65$ & 2.57 & 2.28 & 2.77 & 2.68 & 4.00 & 1.51 & 1.82 \\
\hline $1965-66$ & 1.44 & 1.79 & 1.80 & 2.14 & 2.85 & 1.39 & 1.64 \\
\hline $1966-67$ & 0.81 & 1.08 & 0.88 & 1.06 & 1.64 & 0.73 & 0.94 \\
\hline $1967-68$ & 1.86 & 2.28 & 2.79 & 4.29 & 3.22 & 2.05 & 2.48 \\
\hline $1968-69$ & 1.56 & 1.83 & 2.22 & 2.55 & 2.47 & 0.98 & 1.15 \\
\hline $1969-70$ & 1.15 & 1.13 & 1.47 & 2.23 & 1.13 & 1.72 & 1.92 \\
\hline $1970-71$ & 1.10 & 1.91 & 1.91 & 1.88 & 1.04 & 1.89 & 2.15 \\
\hline $1971-72$ & 1.98 & 2.11 & 1.95 & 2.97 & 1.69 & 2.27 & 2.69 \\
\hline $1972-73$ & 1.43 & 1.71 & 1.95 & 2.37 & 1.39 & 2.06 & 2.53 \\
\hline $1973-74$ & 1.38 & 1.59 & 1.64 & 2.82 & 1.25 & 1.58 & 2.12 \\
\hline $1974-75$ & 1.46 & 1.82 & 1.68 & 3.05 & 1.45 & 1.55 & 2.59 \\
\hline $1975-76$ & 1.69 & 1.88 & 1.79 & 3.02 & 1.42 & 1.29 & 2.23 \\
\hline $1976-77$ & 1.89 & 2.63 & 2.16 & 3.29 & 1.42 & 1.49 & 2.04 \\
\hline $1977-78$ & 3.90 & 4.40 & 3.92 & 4.79 & 3.29 & 1.47 & 2.09 \\
\hline $1978-79$ & 4.44 & 4.95 & 4.89 & 4.99 & 3.90 & 2.21 & 3.13 \\
\hline $1979-80$ & 3.59 & 3.89 & 2.89 & 4.25 & 2.87 & 3.12 & 3.80 \\
\hline $1980-81$ & 3.67 & 4.63 & 4.21 & 5.45 & 3.92 & 3.46 & 4.22 \\
\hline $1981-82$ & 4.27 & 4.29 & 4.01 & 6.23 & 3.58 & 1.92 & 2.80 \\
\hline $1982-83$ & 4.88 & 5.41 & 3.99 & 7.57 & 4.37 & 1.51 & 3.20 \\
\hline $1983-84$ & 5.09 & 6.72 & 5.34 & 5.93 & 4.28 & 2.08 & 4.05 \\
\hline $1984-85$ & 7.30 & 6.88 & 5.59 & 15.91 & 7.08 & 3.02 & 4.84 \\
\hline $1985-86$ & 3.92 & 3.97 & 3.01 & 12.69 & 4.06 & 3.56 & 4.98 \\
\hline $1986-87$ & 4.56 & 6.02 & 3.60 & 10.92 & 3.72 & 4.45 & 5.80 \\
\hline $1987-88$ & 6.72 & 8.73 & 5.69 & 12.99 & 5.58 & 5.35 & 5.93 \\
\hline $1988-89$ & 6.63 & 8.41 & 5.46 & 12.64 & 6.31 & 4.33 & 4.71 \\
\hline $1989-90$ & 6.01 & 6.53 & 5.64 & 15.28 & 5.10 & 5.21 & 6.30 \\
\hline $1990-91$ & 5.38 & 6.58 & 6.31 & 17.10 & 6.11 & 4.59 & 6.85 \\
\hline $1991-92$ & 5.44 & 6.65 & 6.51 & 18.00 & 7.16 & 6.46 & 6.87 \\
\hline $1992-93$ & 3.23 & 3.88 & 2.99 & 13.75 & 3.31 & 2.22 & 3.11 \\
\hline 1993-94 & 3.76 & 4.61 & 2.73 & 9.83 & 2.38 & 3.23 & 3.38 \\
\hline 1994-95 & 3.25 & 4.41 & 3.47 & 11.98 & 2.64 & 2.58 & 1.66 \\
\hline $1995-96$ & 3.62 & 5.57 & 4.44 & 12.59 & 3.63 & 2.14 & 1.77 \\
\hline $1996-97$ & 3.18 & 4.07 & 3.22 & 7.99 & 2.19 & 1.12 & 1.91 \\
\hline $1997-98$ & 2.81 & 4.88 & 3.07 & 8.49 & 1.66 & 0.93 & 1.50 \\
\hline 1998-99 & 4.35 & 5.58 & 5.12 & 12.07 & 4.53 & 1.95 & 2.65 \\
\hline 1999-00 & 3.19 & 4.33 & 2.55 & 6.67 & 2.52 & 3.87 & 3.36 \\
\hline 2000-01 & 2.60 & 4.02 & 2.05 & 6.40 & 1.27 & 2.07 & 2.28 \\
\hline 2001-02 & 2.88 & 4.20 & 2.19 & 7.81 & 2.47 & 1.96 & 2.54 \\
\hline 2002-03 & 2.62 & 3.85 & 2.01 & 8.40 & 2.60 & 1.59 & 2.79 \\
\hline 2003-04 & 2.20 & 3.64 & 1.07 & 7.46 & 7.48 & 1.88 & 3.28 \\
\hline $2004-05^{f}$ & 2.56 & 4.34 & 2.48 & 12.02 & 2.45 & 11.95 & 13.65 \\
\hline
\end{tabular}

${ }^{\mathrm{a}}$ On-tree average price per box (1-3/5 bushel box equivalent) for all methods of sale minus pick and haul charges.

${ }^{b}$ Navel and Hamlin $\quad{ }^{c}$ Parson Brown and Pineapple $\quad{ }^{d}$ Valencia $\quad{ }^{e}$ Marsh (white) or pink Preliminary

Source: Florida Agricultural Statistics Service. 
Table 17-A.--Debt which can be supported per $\$ 1,000.00$ annual payment capacity

\begin{tabular}{|c|c|c|c|c|c|c|c|c|c|c|c|c|c|c|c|}
\hline \multirow{2}{*}{$\begin{array}{c}\text { Loan } \\
\text { term } \\
\text { (years) }\end{array}$} & \multicolumn{15}{|c|}{ Interest rate paid on the loan } \\
\hline & $8.0 \%$ & $8.5 \%$ & $9.0 \%$ & $9.5 \%$ & $10.0 \%$ & $10.5 \%$ & $11.0 \%$ & $11.5 \%$ & $12.0 \%$ & $12.5 \%$ & $13.0 \%$ & $13.5 \%$ & $14.0 \%$ & $14.5 \%$ & $15.0 \%$ \\
\hline 1 & 926 & 922 & 917 & 913 & 909 & 905 & 901 & 897 & 893 & 889 & 885 & 881 & 877 & 873 & 870 \\
\hline 2 & 1,783 & 1,771 & 1,759 & 1,747 & 1,754 & 1,724 & 1,713 & 1,701 & 1,690 & 1,679 & 1,668 & 1,657 & 1,647 & 1,636 & 1,626 \\
\hline 3 & 2,577 & 2,554 & 2,531 & 2,509 & 2,487 & 2,465 & 2,444 & 2,423 & 2,402 & 2,381 & 2,361 & 2,341 & 2,322 & 2,302 & 2,283 \\
\hline 4 & 3,312 & 3,276 & 3,240 & 3,204 & 3,170 & 3,136 & 3,102 & 3,070 & 3,037 & 3,006 & 2,974 & 2,944 & 2,914 & 2,884 & 2,855 \\
\hline 5 & 3,993 & 3,941 & 3,890 & 3,840 & 3,791 & 3,743 & 3,696 & 3,650 & 3,605 & 3,561 & 3,517 & 3,475 & 3,433 & 3,392 & 3,352 \\
\hline 6 & 4,623 & 4,554 & 4,486 & 4,420 & 4,355 & 4,292 & 4,230 & 4,170 & 4,111 & 4,054 & 3,998 & 3,942 & 3,889 & 3,836 & 3,784 \\
\hline 7 & 5,206 & 5,119 & 5,033 & 4,950 & 4,868 & 4,789 & 4,712 & 4,640 & 4,564 & 4,492 & 4,423 & 4,355 & 4,288 & 4,224 & 4,160 \\
\hline 8 & 5,747 & 5,639 & 5,535 & 5,433 & 5,335 & 5,239 & 5,146 & 5,056 & 4,968 & 4,882 & 4,799 & 4,718 & 4,639 & 4,562 & 4,487 \\
\hline 9 & 6,247 & 6,119 & 5,995 & 5,875 & 5,759 & 5,646 & 5,537 & 5,431 & 5,328 & 5,228 & 5,132 & 5,038 & 4,946 & 4,858 & 4,772 \\
\hline 10 & 6,710 & 6,561 & 6,418 & 6,279 & 6,145 & 6,015 & 5,889 & 5,768 & 5,650 & 5,536 & 5,426 & 5,319 & 5,216 & 5,116 & 5,019 \\
\hline 11 & 7,139 & 6,969 & 6,805 & 6,647 & 6,495 & 6,348 & 6,207 & 6,070 & 5,938 & 5,810 & 5,687 & 5,568 & 5,453 & 5,341 & 5,234 \\
\hline 12 & 7,536 & 7,345 & 7,161 & 6,984 & 6,814 & 6,650 & 6,492 & 6,341 & 6,194 & 6,054 & 5,918 & 5,787 & 5,660 & 5,538 & 5,421 \\
\hline 13 & 7,904 & 7,691 & 7,487 & 7,291 & 7,103 & 6,923 & 6,750 & 6,583 & 6,424 & 6,270 & 6,122 & 5,979 & 5,842 & 5,710 & 5,583 \\
\hline 14 & 8,244 & 8,010 & 7,786 & 7,572 & 7,367 & 7,170 & 6,982 & 6,801 & 6,628 & 6,462 & 6,302 & 6,149 & 6,002 & 5,861 & 5,724 \\
\hline$\underline{15}$ & 8,559 & 8,304 & 8,061 & 7,828 & 7,606 & 7,394 & 7,191 & $\underline{6,997^{\mathrm{a}}}$ & 6,811 & 6,633 & 6,462 & 6,299 & 6,142 & 5,992 & 5,847 \\
\hline 16 & 8,851 & 8,576 & 8,313 & 8,062 & 7,824 & 7,596 & 7,379 & 7,172 & 6,974 & 6,785 & 6,604 & 6,431 & 6,265 & 6,106 & 5,954 \\
\hline 17 & 9,122 & 8,825 & 8,543 & 8,276 & 8,022 & 7,779 & 7,549 & 7,329 & 7,119 & 6,920 & 6,729 & 6,547 & 6,373 & 6,207 & 6,048 \\
\hline 18 & 9,372 & 9,056 & 8,756 & 8,471 & 8,201 & 7,945 & 7,702 & 7,470 & 7,250 & 7,040 & 6,840 & 6,649 & 6,467 & 6,294 & 6,128 \\
\hline 19 & 9,603 & 9,268 & 8,950 & 8,650 & 8,365 & 8,095 & 7,839 & 7,596 & 7,366 & 7,146 & 6,938 & 6,739 & 6,551 & 6,370 & 6,198 \\
\hline$\underline{20}$ & 9,818 & 9,463 & 9,129 & 8,812 & 8,514 & 8,231 & 7,963 & $\underline{7,710^{\mathrm{a}}}$ & 7,469 & 7,241 & 7,025 & 6,819 & 6,623 & 6,437 & 6,259 \\
\hline 25 & 10,675 & 10,234 & 9,823 & 9,438 & 9,077 & 8,739 & 8,422 & 8,123 & 7,843 & 7,579 & 7,330 & 7,095 & 6,873 & 6,663 & 6,464 \\
\hline 30 & 11,258 & 10,747 & 10,274 & 9,835 & 9,427 & 9,047 & 8,868 & 8,364 & 8,055 & 7,766 & 7,496 & 7,242 & 7,003 & 6,778 & 6,566 \\
\hline 35 & 11,655 & 11,088 & 10,567 & 10,087 & 9,644 & 9,234 & 8,855 & 8,503 & 8,175 & 7,870 & 7,586 & 7,320 & 7,070 & 6,836 & 6,617 \\
\hline 40 & 11,925 & 11,315 & 10,757 & 10,247 & 9,779 & 9,348 & 8,951 & 8,587 & 8,244 & 7,928 & 7,634 & 7,361 & 7,105 & 6,866 & 6,642 \\
\hline
\end{tabular}

${ }^{a}$ Example. Assumes a $\$ 10,000$ after tax income at $11.5 \%$ interest rate and a 15 -year term mortgage, the total debt which can be supported is $\$ 69,970$ $(\$ 6,997 \times 10)$. At $11.5 \%$ interest rate and a 20 -year term mortgage, the total debt which can be supported is $\$ 77,100(\$ 7,710 \times 10)$. 